\title{
Tree Bark, a Valuable Source of Information on Air Quality
}

\author{
Mariusz Chrabąszcz*, Lucyna Mróz \\ Department of Ecology, Biogeochemistry and Environmental Protection, University of Wrocław, \\ Kanonia 6/8, 50-328 Wrocław, Poland
}

Received: 24 September 2016

Accepted: 17 October 2016

\begin{abstract}
Tree bark has been proven to be a valuable source of information on air pollution. Bark has a large absorbent surface thanks to its deeply furrowed structure. Through the ability to accumulate atmospheric particulate matter (PM), tree bark enables the identification and mapping of organic and inorganic air pollutants. The first study of air quality using tree bark dates back to the second half of the $20^{\text {th }}$ century and such studies are constantly being developed today. Reports published almost every year present the potential of using tree bark of different tree species for monitoring atmospheric pollution. This article presents scientific evidence proving the possibility of using bark in the process of assessing air quality and also describes the main directions of research in this field.
\end{abstract}

Keywords: air pollution, bioindication, air monitoring, tree bark, elemental analysis

\section{Introduction}

Human activity has resulted in an increased release of contaminants into the environment. The atmosphere is the element of the environment that is particularly vulnerable to contamination by substances of human origin [1-5]. Because of continuous mixing of air masses in the atmosphere, contamination can affect areas far from the emission source. In the words of A. Kalinowska (1994), "The wind does not stop at national borders, and pollutants do not spread fairly" [6]. Cross-border transmission of air pollutants makes it impossible for the problem of air quality to be considered in local terms only. Close international cooperation in the monitoring and prevention of air contamination is necessary, and not limited to geographi-

*e-mail: mariusz.chrabaszcz@uwr.edu.pl cal regions or continents, but covering the whole world [2, 7-11].

Air pollutants are substances defined mainly as unnatural constituents of the air and also those naturally occurring in the atmosphere, but at concentrations much higher than standards. Among the contaminants of anthropogenic origin in the air one can distinguish gaseous components and solids (dust) [4]. Gaseous air pollutants include organic and inorganic compounds, e.g., carbon oxides (CO, $\left.\mathrm{CO}_{2}\right)$, sulphur dioxide $\left(\mathrm{SO}_{2}\right)$, nitrogen oxides $\left(\mathrm{NO}_{\mathrm{x}}\right)$, or volatile organic compounds (VOCs), whereas solid air pollutants refer to the particulate matter (PM) in the form of ash, soot, dust, cement, or metallurgical dust. Falling dust that contains, for example, metal oxides permanently contaminates other elements of the environment [7, 1214]. The long-term impact of air pollution consequently results in the impoverishment of biodiversity and reduction of vitality in all ecosystems. Harrison and Yin (2000) 
identified several compounds of airborne PM, possibly toxic to living organisms, including the following: bulk chemical compounds, trace elements, strong acids, and sulphates [7]. A direct correlation between air quality and the incidence of respiratory diseases in humans has been proven $[7-8,15-16]$. Air pollution is considered to be the single largest cause of environmental risk nowadays, and this relationship is also confirmed by the World Health Organization [17]. Although it is assumed that anthropogenic dust represents only $3 \%$ of global PM, the substances introduced by human activity may cause far-reaching and unpredictable changes in the environment [14]. A particular threat to all ecosystems is trace elements, including the heavy metals found in dust pollution. The natural content of metals in the environment is relatively low, so that their excessive emissions into the atmosphere can result in their accumulation in the trophic chain, which in turn contributes to biosphere degradation at every organizational level [18-19].

In order to assess the potential risks arising from air pollution by foreign substances, the most popular are physicochemical tests. Nevertheless, in contrast to physicochemical monitoring that provides information only about air pollution at the time of measurement, biomonitoring using living organisms provides comprehensive data on impurities accumulation in the environment for long periods of time $[10,20]$. Plants are particularly sensitive to changes in the air and are widely used in air monitoring. Assessing air quality based on plants can be done using two methods: analysing impurities accumulated by the plant and using the plant to observe changes (usually unfavourable) in the morphology or physiology of plants caused by pollutants, thus related to ecotoxicology [13, 19-20]. In a study of air quality, lichens and mosses are the most frequently used bioindicators, individual species of which can point directly to the degree of contamination, and special scales have been developed for such species [1, 21-28]. Mosses and lichens may occur in all studied locations, but higher plants are often selected as suitable bioindicators.

Higher plants are not capable of capturing atmospheric pollution as easily as mosses and lichens. Accumulation of air pollution by higher plants is mainly the result of adsorption (deposition on terrestrial parts). The use of higher plant organs in the monitoring of air pollution is practically a form of bioindication consisting mainly of studying the physiological effect of pollution (yellowing, shedding needles and leaves, deformation of limbs, disturbance of flowering or fruiting, loss of bark elasticity) [19-20, 2932]. Among the higher plant organs, leaves have proven to be particularly useful in air monitoring. Since contamination settling on leaves can easily be removed by wind or washed away by precipitation (acid rain can leach alkaline cations), this method does not provide conclusive results regarding air pollution in an area and can even deliver false results [4, 33-35]. Odabasi et al. (2016) distinguished the three most effective pathways of contaminants entering the plant. Besides uptake by the roots, contaminants can penetrate the plants through dry deposition on leaf and absorption through bark surfaces [11]. In recent years, the use of tree bark is becoming more and more popular for determining air pollution results $[4,35]$ This article presents the current state of knowledge regarding the possibility of using tree bark as a bioindicator of historical and cumulative air pollution.

\section{General Structure of Tree Bark}

Bark is commonly known as a uniform cover and the outer layer of trunks of trees and woody shrubs. However, what generally is considered to be bark is in fact an imprecise concept referring only to the outermost parts of its structure. Indeed, bark consists of layers made of a number of tissues of different origin. In the overall structure of so-called bark, there are only two meristematic regions capable of cell division: the cork and the vascular cambium. The outermost, cracked, and irregular outer layer called cork (fellem) is a secondary tissue arising from outward intense divisions of meristematic cells in cork cambium (phellogen). Cork cells are dead, and their cell walls contain suberin, a substance composed of long-chain fatty acids that provide protection against moisture loss, among other things. Inward-dividing phellogen cells result in the formation of a thin layer of live parenchyma cells. This secondary layer of inner cells is called phelloderm. Owing to cambium activity, cork and phelloderm together form a secondary tissue cover called periderm. Besides periderm, in the innermost layers of bark there is secondary phloem emerging from divisions of vascular cambium. The living cells of this non-homogeneous tissue are involved in the transport of organic nutrients. Thus bark, as shown in Fig. 1, consists of two layers: the external periderm layer and the inner layer of secondary phloem [2, 36-37].

Bark is an organ of specific, cracked structure characteristic to the species. As an aboveground part of the plant, it is constantly exposed to weather factors (Fig. 1). The folded structure of bark gives it a relatively high specific surface area. A large surface area of bark with its large sorption capacity makes it able to stably adsorb dust suspended in the air, thus showing even small changes in the environment [2, 4, 26, 29-31, 35, 38-41]. Studies have confirmed that the sorption ability of bark varies significantly, depending on whether it is smooth or wrinkled $[8,13,34]$. Regardless of the physical properties, the factor in favour of the use of bark in monitoring air quality is its wide availability throughout the year. In addition, bark must meet certain criteria to provide clear and comparable measurement results. First of all, bark must be obtained from a species common in the area and having a well-known biology, and thus a large number of samples can be collected. Sampling should be performed in such a way as to be able to obtain clear environmental dependence. Thus, bark samples for research from a given area should be collected from specimens of the same species at a similar age as determined by measuring tree height and circuit diameter at chest height [19-20]. Furthermore, an important argument for using bark in air 


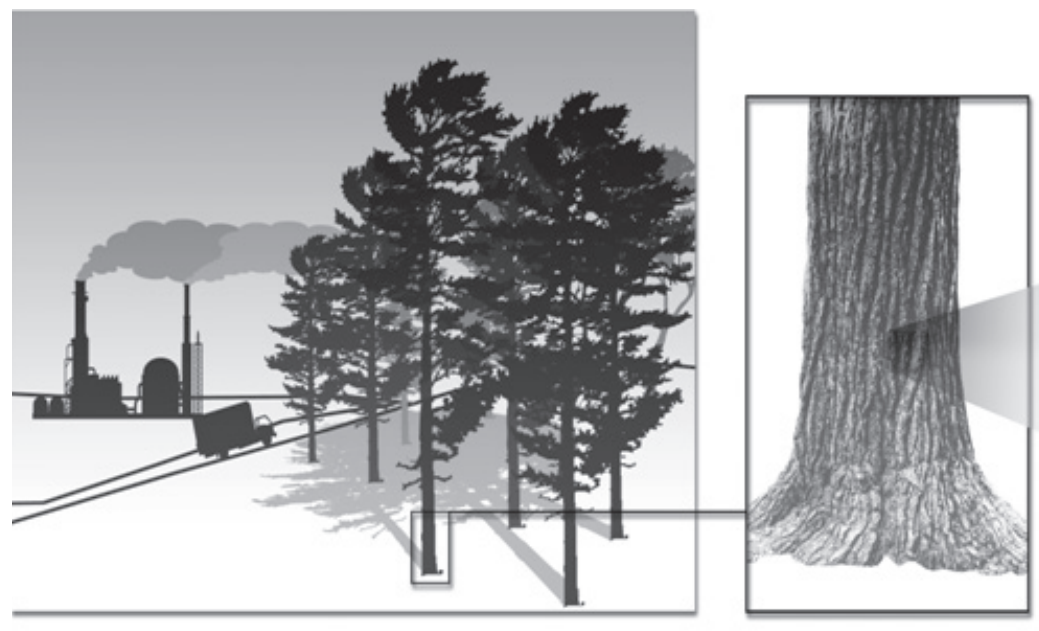

Inner bark

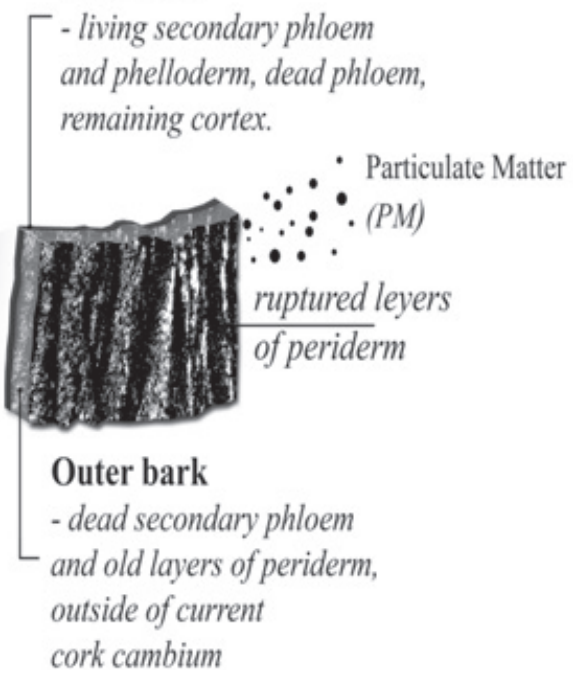

Fig. 1. Tree bark exposure to harmful factors originating mainly from industry and transport; the two-layer structure of the bark makes the impurities contained in the particulate matter deposit only on its outer, dead part.

monitoring is the lack of sampling influence on tree health status [26, 29-30].

\section{Results and Discussion}

\section{Usefulness of the Tree Bark in Monitoring of Air Pollution}

\section{Evaluation of Inorganic Air Pollutants Using Tree Bark}

The first studies on the use of tree bark in monitoring environmental changes were carried out in the second half of the $20^{\text {th }}$ century. The study of air quality conducted on lichens proved that along with lichen samples, tree bark as a substrate should also be collected and treated as a sample. However, initial research was based on the observations of symptoms that the bark of many species showed under the influence of air pollution. The impact of atmospheric pollution is manifested, e.g., in the flaky falling off bark of Fagus sylvatica and Acer pseudoplatanus, and total loss of bark has been recorded with the elm (Ulmus sp.) [20].

The period between the 1960s and the 1980s brought a new line in research of air quality using tree bark. A special contribution in promoting the use of tree bark in air quality monitoring in this period can be attributed to Krystyna Grodzińska, whose work presenting tree bark as a bioindicator of air pollution caused quite a stir in the scientific world. This constituted an assessment of environment acidification by $\mathrm{SO}_{2}$ from transport and industry on the basis of $\mathrm{pH}$ of the plant material tested $[21,29-30,42-43]$. At that time, a relationship was found between $\mathrm{pH}$ measurements of bark extracts and gaseous air pollutants, rich in acidic compounds (e.g., $\mathrm{SO}_{2}$ ), coming from nearby transport routes (traffic pollution) or industrial emitters (industrial heavy metal surveys) [20,
44]. The relationship between the concentration of $\mathrm{SO}_{2}$ in the atmosphere and acidity of the bark of many species was confirmed in studies on bark of such species as Quercus robur, Tilia cordata, and Pinus sylvestris. The results of the research showed, among other things, that the bark of deciduous trees had lower natural acidity than the bark of conifers, making the former potentially more sensitive biomarkers. In subsequent years, further investigation revealed a correlation between $\mathrm{SO}_{2}$ concentration in the atmosphere and sulphur content from the settling dust in bark. Assessment of air pollution based on a study of bark acidity showed the latter to be sensitive only to large amplitude fluctuations in $\mathrm{SO}_{2}$ concentrations in the air. On the other hand, determination of electrolytic conductivity of bark extracts proved to be a much more sensitive method even with small changes in $\mathrm{SO}_{2}$ and $\mathrm{CO}_{2}$ emissions into the atmosphere [20, 31, 43-44]. Notwithstanding, determining bark acidity is a still popular and inexpensive method for studying air pollution in areas subjected to strong human pressure. Studies conducted by Stainor et al. (2011) on the acidity of a four-species bark, including reports on black locust and European yew [45] as well as horseradish (2015) [32] that expand on the results of Grodzińska $(1977,1979)$ regarding the $\mathrm{pH}$ value of Scots pine bark in response to environmental conditions near Krakow [29$30]$, confirmed the possibility of the use of acidity of tree bark as an indicator of air pollution by acidic oxides of anthropogenic origin.

\section{Elemental Analysis of Tree Bark}

Apart from using tree bark to monitor changes in "typical" air pollutants $\left(\mathrm{SO}_{2}, \mathrm{NO}_{\mathrm{x}}\right)$, another group of researchers began to focus on the evaluation of the content in bark of heavy metals emitted by anthropogenic sources $[1,20]$. The idea of holistic multi-elemental analysis of the plant material dates back to the turn of the 1970s and 1980s, 
when it was noted that a linear relationship between the content of elements in the plant material and their contents in the environment existed [43, 46-47]. Initially, in studies involving bark, attention was focused only on impurities adsorbed on its surface in bark pockets. It was thought that adsorption on the bark surface (on dead plant material) was the only method of air pollution accumulation. It is now known that the analysis of element content in bark provides information about the levels of both air and soil pollution $[20,33-34,39,48]$. This is because bark consists of the outer (dead) part, passively adsorbing air pollution, and the internal (live) part, absorbing pollution through the root system, straight from the soil (Fig. 1). Moreover, the elements can be sorted with increasing capacity to move from roots to shoots. Metals such as $\mathrm{Cu}$ and $\mathrm{Pb}$ show much lower mobility of this matter than $\mathrm{Tl}$ and $\mathrm{Cd}$. On this basis it can be inferred that the large average content of less mobile elements $(\mathrm{Co}, \mathrm{Cr}, \mathrm{Cu}$ and $\mathrm{Pb})$ in tree bark may come from air pollution. On the contrary, the source of bark pollution caused by highly mobile metals $(\mathrm{Cd}$ and $\mathrm{Zn}$ ) is more difficult to identify unambiguously [18, 33$34,49]$. The two-layer structure of tree bark causes a lot of problems with the interpretation of results. Since the contribution of the inner part of bark to the total content of elements in bark is relatively small, it has been neglected by most authors [2, 4]. However, recent studies suggest a significant gradient of concentration of the elements within the layers of the bark. Janta et al. (2016) reported that concentrations of some metals, including $\mathrm{Cr}, \mathrm{Cu}, \mathrm{Pb}$, and $\mathrm{Zn}$, were highest in the cork layer of Cassia fistula bark, decreased in the second and third layers (phloem), and increased in the innermost layer (vascular cambium) [37]. Similar patterns of content gradients were observed for $\mathrm{Hg}$ in Pinus nigra bark [41].

It can be noted that since the late 1970s [46-47], elemental analysis of tree bark has been the primary direction in the study of air quality based on tree bark as a biological material. Almost every year scientific reports present the possibility of using the bark of other trees, previously not described for biomonitoring, in the effective monitoring of trace elements released into the atmosphere. Scientific reports predominantly refer to determination of the elemental composition of tree bark from urban agglomerations (both traffic and agglomeration air pollution) [4, $11,26,31,33-34,38-40,46-58]$, but also from industrial emitters (industrial air pollution) [2, 8, 13, 59]. Multi-elemental analysis of the tree bark was recently used for determining air quality in Brazil in urban areas of Paranaguá [10] and São Paulo [60], Chiang Mai in northern Thailand [37], and in Italy's Liguria region [9, 35]. Considering pollution from industrial emitters, metallurgical industry sites are particularly troublesome "hotspots" of pollution emission into the atmosphere. Metals accumulate in the environment, which causes their practical non-degradable character. Bellis et al. (2003) proved that the bark of Japanese oaks can be suitable for monitoring the historical change in atmospheric arsenic deposition in the vicinity of industrial sites [61]. Also in the areas of mining, recent studies of Chiarantini et al. (2016) have shown that black pine bark can be successfully used to determine air pollution by mercury compounds [41]. Research conducted by Conte et al. (2016) confirmed the possibility of using the bark of the Slippery Elm tree to detect uranium from nuclear fuel [14].

Especially valuable information on the possibility of using tree bark in bioindication was provided by studies conducted on large areas, countries, or geographical regions. Among the extensive research on the elemental composition of tree bark the work of Wolterbeek et al. (1996) conducted throughout the entire Netherlands (123 locations) regarding oak bark [62], among other materials, should particularly be noted, as should the study that Bohm et al. (1998) carried out in the western Czech Republic (457 locations) concerning multi-elemental analysis of oak bark [1]. Extensive research was conducted in Germany by Schulz et al. (2000) on the bark of Scots pine (273 locations) [63-64] and in Portugal by Pacheco et al. $(2001,2002)$ on the bark of olive trees (28 sampling sites) [65-66] and plane tree (2008) transplants in a mixed environment [65]. Saarela at al. (2005) [68] conducted multi-elemental analysis on the bark of birch, spruce, and Scots pine in southwestern Finland, a reference to the research carried out almost 10 years earlier by Poikolainen (1997) [44].

Tree bark as a biological material contains in its structure many trace elements whose content is variable in time and can therefore act as a selective filter for some elements, artificially increasing or decreasing the contents. The natural content of elements in the cortex, referred to as the bark matrix, can affect the results [5, 20, 39, 55]. Studies of Cantion et al. $(2009,2012)$ on the anthropological fraction of impurities deposited on bark show that bark may indeed respond as a selective filter, increasing or excluding certain elements, e.g. Ca, K, or Fe [33-34]. Also, studies on the adsorption ability of maritime pine bark confirm that the bark can act as a selective filter of heavy metals [55]. Therefore, in order to determine the influence of actual environmental pollution based on the elemental composition of tree bark, the natural level of elements in bark must be taken into account with awareness of possible interactions between the elements in bark and in dust. Furthermore, the elemental composition of bark also depends on the dryness of wet deposition. In contrast to studies based on leaves and other above-ground plant organs, the location of bark effectively prevents rainfall from washing away impurities absorbed on the surface $[2$, 5, 13, 33-34, 39, 63].

Although the bark of trees is a good natural sorbent of air pollution rich in trace elements, assessment of air quality should not be based only on studying the elemental composition of bark. Many trace elements present in air pollution (such as $\mathrm{Al}, \mathrm{Fe}, \mathrm{Cr}, \mathrm{Ni}$, or $\mathrm{As}$ ) may both derive from natural sources and be emitted through human activity. The method of bark elemental analysis does not enable us to fully distinguish between natural and anthropogenic pollution and, even more, using this method it is not possible to distinguish between various sources among anthropogenic emissions [1, 8, 52-53]. Recent studies with 
pine bark suggest the possibility of using lanthanide (La, $\mathrm{Ce}, \mathrm{Pr}, \mathrm{Nd}, \mathrm{Sm}, \mathrm{Gd}, \mathrm{Dy}, \mathrm{Er}, \mathrm{Yb}$ ) to investigate industrial emissions [11]. However, a newer research approach to identifying contaminants and their emitters is to determine isotope ratios in the studied materials [27, 38]. Isotopic studies can be used to determine the origin of the elements in the atmosphere. Due to increased transport and industrial development, $\mathrm{Sr}, \mathrm{Nd}, \mathrm{Pb}$, and $\mathrm{U}$ isotopes are of particular importance in environmental studies, also successfully determined in tree bark [39, $53,69]$. Also, stable isotope ratio analysis of $\mathrm{C}, \mathrm{N}$, and $\mathrm{O}\left({ }^{13} \mathrm{C},{ }^{18} \mathrm{O}\right.$, and $\left.{ }^{15} \mathrm{~N}\right)$ can be successfully used as longterm indicators of environmental changes according to Cocozza et al. (2016) [5]. Recent studies related to the content of the isotope concerned ${ }^{137} \mathrm{Cs}$ in the bark of mainly oak and spruce. ${ }^{137} \mathrm{Cs}$ in the atmosphere comes from nuclear tests and also from the 1986 nuclear disaster in Chernobyl. Studies have demonstrated the highest content of ${ }^{137} \mathrm{Cs}$ in oak bark, which can be a promising long-term indicator of contamination of this isotope [70]. Apart from isotopes, tree bark may also provide information relating to element speciation and degree of oxidation. Studies of Mandiwana et al. (2006) demonstrated the possibility of using acacia bark as a bioindicator of toxic hexavalent chromium Cr (VI) in air pollution in South Africa due to the high accumulating ability of bark for chromium from air dust [59].

\section{Evaluation of Air Organic Pollutants Using Tree Bark}

In terms of chemical structure, pollutants in the atmosphere can be divided into organic and inorganic compounds [7]. Inorganic contaminants are represented by oxides and heavy metals described above. Organic contaminants are a fixed group composed of substances that can easily pass in the form of vapour or gas and have low water solubility. The general name of organic compounds in the atmosphere is volatile organic compounds (VOCs). These compounds, such as polycyclic aromatic hydrocarbons, polychlorinated biphenyls, organobromine compounds, or dioxins, include, besides the carbon backbone chain, oxygen, hydrogen, fluorine, chlorine, sulphur, nitrogen, or bromine in their structure. Many organic pollutants are dangerous to the environment due to their resistance to degradation in natural conditions, hence the term POPs (persistent organic pollutants). Organic compounds from anthropogenic sources are never present in the environment individually, but always as multicomponent mixtures of varying compositions. Many of the organic compounds emitted into the environment have a detrimental impact on the biosphere. Toxicity and even certain carcinogenicities of some of them have been proven. It is still not fully known how they interact in mixtures with other types of pollutants. Therefore, monitoring POPs emitted into the environment seems to be extremely important $[12,25,50,71-81]$.

Studies of organic pollutants from the air using bark were initially limited to the determination of polyaromat- ic hydrocarbons. PAHs are a large organic group of compounds that occur in both gas phase and solid air pollution. Although about 100 different PAHs are known, only 17 are present in the environment - with at least two fused aromatic rings being the basis of their chemical structure. Many PAHs exert toxic and even carcinogenic effects on humans, e.g., benzo(a)pyrene. The study of air quality based on, among other things, pine bark [25, 74, 76] or camphor [77] in both urban and industrial environments has proven that tree bark can be successfully used to assess the spatial distribution of ambient air PAHs. In a study by Rauert and Harner (2016), authors analyzed the bark of 5-15-year-old red pines and divided PAHs into fractions: alkylated-PAHs, nitro, and oxy-PAHs. The results showed a trend of spreading of POPs confirming the usefulness of the bark to evaluate historical air pollution [81]. Furthermore, bark is able to capture PAHs from the air as well as from commercial filters, e.g., polyurethane foam samplers (PUFs), which is confirmed by a study of Li et al. (2016) [80].

Relatively recently, tree bark has been used as a passive air indicator for organic compounds, including dioxins (e.g., polychlorinated dibenzofurans, PCDFs, or dibenzo-p-dioxins, PCDDs), polychlorinated biphenyls (PCBs), brominated flame retardants (BFRs), chlorinated pesticides, and ammonium nitrate $[25,58,73,78,82-$ 83]. Research conducted by Gueguen et al. (2011) [12] as well as Salamova and Hites (2012) [84] indicates that tree bark is a particularly sensitive indicator of organobromine compounds: PBDEs (polybrominated diphenyl ethers) and PCBs. However, having a long life cycle, tree bark consequently can accumulate pollutants over a significant amount of time. Hermanson and Johnson (2007) report that due to the presence of certain lipids in structure, bark is able to act as a passive accumulator of organic compounds in the air over the life of the tree [73]. Nevertheless, according to Zhou et al. (2014, 2015), tree bark should be used as an indicator of atmospheric organic contaminants only in some situations because, considering pollution by organic substances, bark is not suitable for determining precise changes in the atmosphere [77-78]. Studies of Wang et al. (2015) carried out over four seasons of the year confirm that tree bark can be an indicator of short-chain chlorinated paraffins, another group of POPs (SCCPs) [58]. The exact qualitative and quantitative analysis of PCBs, carried out on samples of bark from the area of the former production site in Sauget, Illinois (USA), confirmed the high correlation between proximity to the place of PCB emissions and the content in the bark of trees persisting even after several years of cessation of emissions [79, 82]. Thus, tree bark is able to reveal the actual state of air pollution and topsoil by organic substances.

There is no doubt that the analysis for the content of organic compounds of atmospheric origin is currently the main direction of research on the bark of trees as a bioindicator. The usefulness of tree bark for determining atmospheric POPs distribution has been proven experimentally, e.g., in extensive research in China [50, 58, 72, 77-78], 
Table 1. Alphabetic list of selected tree species whose bark has been used in studies of air quality along with chronologically arranged references.

\begin{tabular}{|c|c|c|c|c|}
\hline Genus: & Species: & Determined parameters: & Location: & Reference: \\
\hline Acacia & Acacia karroo & $\mathrm{Cr}(\mathrm{VI})$ & $\begin{array}{l}\text { Northwest } \\
\text { South Africa }\end{array}$ & $\begin{array}{l}\text { Mandiwana K.L. et } \\
\text { al., } 2006 \text { [59] }\end{array}$ \\
\hline \multirow{6}{*}{ Acer } & \multirow{2}{*}{$\begin{array}{c}\text { Acer } \\
\text { platanoides }\end{array}$} & $\mathrm{pH}\left(\mathrm{SO}_{2}\right)^{*}$, buffer capacity & Stockholm (Sweden) & Skye E. 1968 [21] \\
\hline & & $\mathrm{pH}\left(\mathrm{SO}_{2}\right), \mathrm{Ca}, \mathrm{K}, \mathrm{Na}$ & $\begin{array}{l}\text { Frankfurt am Main, Hesse } \\
\text { (Germany) }\end{array}$ & $\begin{array}{l}\text { Lötschert W. and } \\
\text { Köhm H.J. } 1977 \text { [46] }\end{array}$ \\
\hline & \multirow{4}{*}{$\begin{array}{c}\text { Acer } \\
\text { pseudoplatanus }\end{array}$} & $\mathrm{pH}\left(\mathrm{SO}_{2}\right)$, buffer capacity & Stockholm (Sweden) & Skye E. 1968 [21] \\
\hline & & ${ }^{355} \mathrm{U} /{ }^{238} \mathrm{U}$ ratio & $\begin{array}{c}\text { North Wales, } \\
\text { northeast Derbyshire, Scilly } \\
\text { Isles, Drax in Yorkshire (UK) }\end{array}$ & $\begin{array}{l}\text { Bellis D.J. et al., } \\
\quad 2001[38]\end{array}$ \\
\hline & & $\begin{array}{c}\mathrm{Ag}, \mathrm{Al}, \mathrm{As}, \mathrm{Ba}, \mathrm{Cd}, \mathrm{Co}, \mathrm{Cr}, \mathrm{Cu}, \mathrm{Fe}, \mathrm{Mn}, \\
\mathrm{Ni}, \mathrm{Pb}, \mathrm{Sb}, \mathrm{Se}, \mathrm{Sn}, \mathrm{Ti}, \mathrm{V}, \mathrm{Zn}\end{array}$ & Sheffield (United Kingdom) & $\begin{array}{l}\text { Schelle E. et al., } 2008 \\
{[8]}\end{array}$ \\
\hline & & $\mathrm{pH}\left(\mathrm{SO}_{2}\right)$ & $\begin{array}{l}\text { Cities of Upper Silesian } \\
\text { Industrial Region (Poland) }\end{array}$ & $\begin{array}{l}\text { Steindor K. et al., } \\
2010[45]\end{array}$ \\
\hline \multirow[b]{2}{*}{ Aesculus } & \multirow{2}{*}{$\begin{array}{c}\text { Aesculus } \\
\text { hippocastanum }\end{array}$} & $\mathrm{pH}\left(\mathrm{SO}_{2}\right)$, buffer capacity & Stockholm (Sweden) & Skye E. 1968 [21] \\
\hline & & $\mathrm{Cd}, \mathrm{Cu}, \mathrm{Pb}, \mathrm{Zn}$ & $\begin{array}{l}\text { Edirne, Edirne Province } \\
\text { (Turkey) }\end{array}$ & $\begin{array}{l}\text { Yilmaz R. et al., } 2006 \\
\text { [48] }\end{array}$ \\
\hline \multirow[b]{2}{*}{ Alnus } & Alnus glutinosa & \multirow[b]{2}{*}{$\mathrm{pH}\left(\mathrm{SO}_{2}\right)$, buffer capacity } & Stockholm (Sweden) & Skye E. 1968 [21] \\
\hline & Alnus incana & & $\begin{array}{l}\text { Białowieża National Park, } \\
\text { Niepołomice Forest (Poland) }\end{array}$ & $\begin{array}{c}\text { Grodzińska K. } 1977 \\
{[29]}\end{array}$ \\
\hline \multirow{3}{*}{ Betula } & \multirow[b]{2}{*}{ Betula pendula } & $\mathrm{pH}\left(\mathrm{SO}_{2}\right)$, buffer capacity & Stockholm (Sweden) & Skye E. 1968 [21] \\
\hline & & $\begin{array}{c}\mathrm{Ba}, \mathrm{Ca}, \mathrm{Cd}, \mathrm{Cu}, \mathrm{Fe}, \mathrm{K}, \mathrm{Mn}, \mathrm{Ni}, \mathrm{P}, \mathrm{Pb} \\
\mathrm{Rb}, \mathrm{S}, \mathrm{Sr}, \mathrm{Zn}\end{array}$ & Southwest Finland & $\begin{array}{l}\text { Saarela K.E. et al., } \\
2005[68]\end{array}$ \\
\hline & $\begin{array}{c}\text { Betula } \\
\text { pubescens }\end{array}$ & $\mathrm{pH}\left(\mathrm{SO}_{2}\right)$, buffer capacity & Stockholm (Sweden) & Skye E. 1968 [21] \\
\hline Caesalpinia & $\begin{array}{l}\text { Caesalpinia } \\
\text { pluviosa }\end{array}$ & $\begin{array}{c}\mathrm{Al}, \mathrm{Ba}, \mathrm{Ca}, \mathrm{Cl}, \mathrm{Cu}, \mathrm{Fe}, \mathrm{K}, \mathrm{Mg}, \mathrm{Mn}, \mathrm{Na} \\
\text { P, Rb, S, Sr, Zn }\end{array}$ & $\begin{array}{c}\text { São } \\
\text { Paulo (Brasil) }\end{array}$ & $\begin{array}{l}\text { Moreira T.C.L. et al., } \\
2016[60]\end{array}$ \\
\hline Carpinus & $\begin{array}{l}\text { Carpinus } \\
\text { betulus }\end{array}$ & $\mathrm{pH}\left(\mathrm{SO}_{2}\right)$, buffer capacity & $\begin{array}{l}\text { Białowieża National Park, } \\
\text { Niepołomice Forest (Poland) }\end{array}$ & $\begin{array}{c}\text { Grodzińska K. } 1977 \\
{[29]}\end{array}$ \\
\hline Cassia & Cassia fistula & $\mathrm{Cr}, \mathrm{Cu}, \mathrm{Fe}, \mathrm{Pb}, \mathrm{Zn}$ & Chiang Mai (Thailand) & $\begin{array}{c}\text { Janta R. et al., } 2016 \\
{[37]}\end{array}$ \\
\hline Cedrus & $\begin{array}{l}\text { Cedrus } \\
\text { deodara }\end{array}$ & PCDD/Fs, PBDEs, PCBs & Luqiao (China) & $\begin{array}{l}\text { Wen S. et al., } 2009 \\
{[50]}\end{array}$ \\
\hline \multirow{2}{*}{ Cinnamomum } & \multirow{2}{*}{$\begin{array}{l}\text { Cinnamomum } \\
\text { camphora }\end{array}$} & PAHs & \multirow{2}{*}{ Southern Jiangsu, (China) } & $\begin{array}{c}\text { Zhou L. et al., } 2014 \\
{[77]}\end{array}$ \\
\hline & & PCBs, PBDEs & & $\begin{array}{l}\text { Zhou L. et al., } 2015 \\
\text { [78] }\end{array}$ \\
\hline Coryllus & $\begin{array}{l}\text { Coryllus } \\
\text { avellana }\end{array}$ & $\mathrm{pH}\left(\mathrm{SO}_{2}\right)$, buffer capacity & $\begin{array}{l}\text { Białowieża National Park, } \\
\text { Niepołomice Forest, (Poland) }\end{array}$ & $\begin{array}{c}\text { Grodzińska K. } 1977 \\
\text { [29] }\end{array}$ \\
\hline Cupressus & $\begin{array}{c}\text { Cupressus } \\
\text { semervirens }\end{array}$ & $\begin{array}{c}\mathrm{pH}\left(\mathrm{SO}_{2}\right) ; \\
\mathrm{Cd}, \mathrm{Co}, \mathrm{Cu}, \mathrm{Cr}, \mathrm{Fe}, \mathrm{Mn}, \mathrm{Ni}, \mathrm{Pb}, \mathrm{Zn}\end{array}$ & Amman (Jordan) & $\begin{array}{l}\text { El-Hasan T. et al., } \\
2002[19]\end{array}$ \\
\hline \multirow{4}{*}{ Fagus } & \multirow{3}{*}{ Fagus sylvatica } & $\mathrm{pH}\left(\mathrm{SO}_{2}\right)$, electrolytic conductivity & Navarra (Spain) & $\begin{array}{l}\text { Santamaria J.M. and } \\
\text { Martin A. } 1997 \text { [31] }\end{array}$ \\
\hline & & ${ }^{35} \mathrm{U} /{ }^{238} \mathrm{U}$ ratio & $\begin{array}{c}\text { North Wales, } \\
\text { northeast Derbyshire, Scilly } \\
\text { Isles, Drax in Yorkshire } \\
\text { (United Kingdom) }\end{array}$ & $\begin{array}{l}\text { Bellis D.J. et al., } \\
2001[38]\end{array}$ \\
\hline & & $\begin{array}{c}\mathrm{As}, \mathrm{Cd}, \mathrm{Co}, \mathrm{Cr}, \mathrm{Cu}, \mathrm{Fe}, \mathrm{Mn}, \mathrm{Mo}, \mathrm{Ni}, \\
\mathrm{Pb}, \mathrm{Sb}, \mathrm{Sn}, \mathrm{V}, \mathrm{Zn} ; \\
\mathrm{PCBs}, \mathrm{PCDD} / \mathrm{Fs}\end{array}$ & $\begin{array}{l}\text { Strasburg (France) } \\
\text { Kehl (Germany) }\end{array}$ & $\begin{array}{l}\text { Guéguen F. et al., } \\
2011 \text { [12] }\end{array}$ \\
\hline & $\begin{array}{c}\text { Fagus } \\
\text { longipetiolata }\end{array}$ & PAHs & Southeast China & $\begin{array}{l}\text { Wang et al., } 2004 \\
\text { [72] }\end{array}$ \\
\hline
\end{tabular}


Table 1. Continued.

\begin{tabular}{|c|c|c|c|c|}
\hline \multirow{6}{*}{ Fraxinus } & \multirow{6}{*}{$\begin{array}{l}\text { Fraxinus } \\
\text { excelsior }\end{array}$} & \multirow[b]{2}{*}{$\mathrm{pH}\left(\mathrm{SO}_{2}\right)$, buffer capacity } & Stockholm (Sweden) & Skye E. 1968 [21] \\
\hline & & & $\begin{array}{c}\text { Białowieża National Park, } \\
\text { Niepołomice Forest, (Poland) }\end{array}$ & $\begin{array}{c}\text { Grodzińska K. } 1977 \\
{[29]}\end{array}$ \\
\hline & & $\mathrm{pH}\left(\mathrm{SO}_{2}\right), \mathrm{Cd}, \mathrm{Mn}, \mathrm{Ni}, \mathrm{Pb}$ & $\begin{array}{c}\text { Frankfurt am Main, Hesse } \\
\text { (Germany) }\end{array}$ & $\begin{array}{c}\text { Lötschert W. and } \\
\text { Köhm H.J. } 1977 \text { [46] }\end{array}$ \\
\hline & & $\begin{array}{c}\mathrm{Al}, \mathrm{As}, \mathrm{Ba}, \mathrm{Ca}, \mathrm{Ce}, \mathrm{Cd}, \mathrm{Co}, \mathrm{Cr}, \mathrm{Cs}, \mathrm{Cu} \\
\mathrm{Fe}, \mathrm{Ga}, \mathrm{Hf}, \mathrm{K}, \mathrm{La}, \mathrm{Mg}, \mathrm{Mn}, \mathrm{Mo}, \mathrm{Na} \\
\mathrm{Ni}, \mathrm{Pb}, \mathrm{Rb}, \mathrm{Sb}, \mathrm{Si}, \mathrm{Sm}, \mathrm{Sn}, \mathrm{Sr}, \mathrm{Ti}, \mathrm{U}, \mathrm{V} \\
\text { W, Zn, Zr }\end{array}$ & Grenoble (France) & $\begin{array}{l}\text { Catinon M. et al., } \\
2009 \text { [33] }\end{array}$ \\
\hline & & $\mathrm{pH}\left(\mathrm{SO}_{2}\right)$ & $\begin{array}{l}\text { Cities of Upper Silesian } \\
\text { Industrial Region (Poland) }\end{array}$ & $\begin{array}{l}\text { Steindor K. et al., } \\
2010[45]\end{array}$ \\
\hline & & $\begin{array}{c}\text { Al, As, Ba, Ca, Cd, Co, Cr, Cu, Fe, K, } \\
\mathrm{La}, \mathrm{Mg}, \mathrm{Mn}, \mathrm{Mo}, \mathrm{Ni}, \mathrm{U}, \mathrm{Pb}, \mathrm{Rb}, \mathrm{Sb}, \mathrm{Se} \\
\text { Si, Th, Ti, U, V, Zn }\end{array}$ & Saint-Martin-d'Hères (France) & $\begin{array}{l}\text { Catinon M. et al., } \\
2012[34]\end{array}$ \\
\hline Moringa & $\begin{array}{l}\text { Moringa } \\
\text { oleifera }\end{array}$ & $\begin{array}{c}\text { As, } \mathrm{Ca}, \mathrm{Co}, \mathrm{Cr}, \mathrm{Cu}, \mathrm{Cd}, \mathrm{Fe}, \mathrm{K}, \mathrm{Mg} \\
\text { Mn, Ni, Pb, Zn }\end{array}$ & $\begin{array}{l}\text { Agra, Aligarh } \\
\text { (India) }\end{array}$ & $\begin{array}{l}\text { Gupta J. et al., } 2014 \\
{[56]}\end{array}$ \\
\hline Olea & Olea europaea & $\begin{array}{l}46 \text { elements including: } \mathrm{Al}, \mathrm{As}, \mathrm{Cl}, \mathrm{Cu} \text {, } \\
\text { Fe, } \mathrm{La}, \mathrm{Mn}, \mathrm{Na}, \mathrm{Sc}, \mathrm{Ti}\end{array}$ & Northwest Portugal & $\begin{array}{l}\text { Pacheco A.M.G et al., } \\
\text { 2001, } 2002[65,66] .\end{array}$ \\
\hline Parkia & $\begin{array}{c}\text { Parkia } \\
\text { biglobosa }\end{array}$ & $\mathrm{Cd}, \mathrm{Cr}, \mathrm{Cu}, \mathrm{Mn}, \mathrm{Ni}, \mathrm{Pb}, \mathrm{Zn}$ & North-central Nigeria & $\begin{array}{l}\text { Olatunji O.S. et al., } \\
2016[13]\end{array}$ \\
\hline \multirow{3}{*}{ Picea } & Picea sp. & $\mathrm{pH}\left(\mathrm{SO}_{2}\right)$, buffer capacity & Stockholm (Sweden) & Skye E. 1968 [21] \\
\hline & \multirow{2}{*}{ Picea abies } & $\begin{array}{c}\mathrm{Ba}, \mathrm{Ca}, \mathrm{Cd}, \mathrm{Cu}, \mathrm{Fe}, \mathrm{K}, \mathrm{Mn}, \mathrm{Ni}, \mathrm{P}, \mathrm{Pb} \\
\mathrm{Rb}, \mathrm{S}, \mathrm{Sr}, \mathrm{Zn}\end{array}$ & Southwest Finland & $\begin{array}{l}\text { Saarela K.E. et al., } \\
2005 \text { [68] }\end{array}$ \\
\hline & & ${ }^{137} \mathrm{Cs}$ & $\begin{array}{l}\text { Northwest, southwest, and } \\
\text { central Romania }\end{array}$ & $\begin{array}{c}\text { Cosma C. et al., } 2016 \\
{[70]}\end{array}$ \\
\hline \multirow{11}{*}{ Pinus } & \multirow[b]{2}{*}{ Pinus sp. } & $\mathrm{pH}\left(\mathrm{SO}_{2}\right)$, buffer capacity & Stockholm (Sweden) & Skye E. 1968 [21] \\
\hline & & SCCPs & Beijing (China) & $\begin{array}{l}\text { Wang T. et al., } 2015 \\
\text { [58] }\end{array}$ \\
\hline & \multirow{2}{*}{ Pinus brutia } & PAHs & $\begin{array}{l}\text { Iskenderun, Hatay Province } \\
\text { (Turkey) }\end{array}$ & $\begin{array}{l}\text { Falay E.O. et al., } \\
2013 \text { [76] }\end{array}$ \\
\hline & & $\begin{array}{l}48 \text { elements including lanthanides (La, } \\
\text { Ce, Pr, Nd, Sm, Gd, Dy, Er, Yb) }\end{array}$ & $\begin{array}{l}\text { Aliağa, Izmir Province } \\
\text { (Turkey) }\end{array}$ & $\begin{array}{l}\text { Odabasi M. et al., } \\
2016[11]\end{array}$ \\
\hline & \multirow{7}{*}{ Pinus sylvestris } & $\mathrm{pH}\left(\mathrm{SO}_{2}\right)$, buffer capacity & $\begin{array}{c}\text { Białowieża National Park, } \\
\text { Niepołomice Forest, (Poland) }\end{array}$ & $\begin{array}{c}\text { Grodzińska K. 1977, } \\
1979[29,30] \\
\end{array}$ \\
\hline & & $\begin{array}{c}\mathrm{pH}\left(\mathrm{SO}_{2}\right) \text { electrolytic conductivity; } \\
\mathrm{Cd}, \mathrm{Co}, \mathrm{Cr}, \mathrm{Cu}, \mathrm{Fe}, \mathrm{Mn}, \mathrm{Ni}, \mathrm{Pb}, \mathrm{Ti}, \mathrm{V} \\
\mathrm{Zn}\end{array}$ & $\begin{array}{l}\text { Northern Finland, } \\
\text { Kola Peninsula (Russia) }\end{array}$ & $\begin{array}{l}\text { Poikolainen J. } 1997 \\
\text { [44] }\end{array}$ \\
\hline & & $\mathrm{pH}\left(\mathrm{SO}_{2}\right)$, electrolytic conductivity & Navarra (Spain) & $\begin{array}{l}\text { Santamaria J.M. and } \\
\text { Martin A. } 1997 \text { [31] }\end{array}$ \\
\hline & & $\begin{array}{c}\mathrm{Al}, \mathrm{As}, \mathrm{B}, \mathrm{Ca}, \mathrm{Cd}, \mathrm{Ce}, \mathrm{Cr}, \mathrm{Cu}, \mathrm{Fe}, \mathrm{Hg} \text {, } \\
\mathrm{La}, \mathrm{Mo}, \mathrm{Nd}, \mathrm{Ni}, \mathrm{Pb}, \mathrm{Sr}, \mathrm{Ti}, \mathrm{V}, \mathrm{W}, \mathrm{Zr} \text {, } \\
\mathrm{Zn} \text {; } \\
\mathrm{NH}_{4}^{+}, \mathrm{NO}_{3}^{+}, \mathrm{PO}_{4}^{+3}, \mathrm{SO}_{4}^{+2} \text {; } \\
\text { PAHs, a-hexachlorocyclohexane (a- } \\
\mathrm{HCH} \text { ) and dichlorodiphenyltrichloro- } \\
\text { ethane (DDT) }\end{array}$ & $\begin{array}{l}\text { Throughout Germany, also } \\
\text { areas in Denmark, Norway, } \\
\text { Poland, Russia }\end{array}$ & $\begin{array}{c}\text { Schulz Z. et al., } 2000 \\
{[63,64]}\end{array}$ \\
\hline & & ${ }^{35} \mathrm{U} / 238 \mathrm{U}$ ratio. & $\begin{array}{c}\text { North Wales, } \\
\text { northeast Derbyshire, Scilly } \\
\text { Isles, Drax in Yorkshire (UK) }\end{array}$ & $\begin{array}{l}\text { Bellis D.J. et al., } \\
2001[38]\end{array}$ \\
\hline & & $\begin{array}{l}\mathrm{Cd}, \mathrm{Cu}, \mathrm{Hg}, \mathrm{Pb}, \mathrm{S}, \mathrm{Zn} ; \\
\text { PAHs, phenols }\end{array}$ & $\begin{array}{c}\text { Świętokrzyskie Mountains } \\
\text { (Poland) }\end{array}$ & $\begin{array}{c}\text { Migaszewski Z. et al., } \\
2002[25] \\
\end{array}$ \\
\hline & & $\begin{array}{c}\mathrm{Ba}, \mathrm{Ca}, \mathrm{Cd}, \mathrm{Cu}, \mathrm{Fe}, \mathrm{K}, \mathrm{Mn}, \mathrm{Ni}, \mathrm{P}, \mathrm{Pb} \\
\mathrm{Rb}, \mathrm{S}, \mathrm{Sr}, \mathrm{Zn}\end{array}$ & Southwest Finland & $\begin{array}{l}\text { Saarela K.E. et al., } \\
2005 \text { [68] }\end{array}$ \\
\hline
\end{tabular}


Table 1. Continued.

\begin{tabular}{|c|c|c|c|c|}
\hline \multirow{13}{*}{ Pinus } & \multirow{5}{*}{ Pinus sylvestris } & $\mathrm{Al}, \mathrm{Cd}, \mathrm{Cr}, \mathrm{Cu}, \mathrm{Fe}, \mathrm{Mn}, \mathrm{Ni}, \mathrm{Pb}, \mathrm{Zn}$ & Stalowa Wola (Poland) & $\begin{array}{c}\text { Samecka-Cymerman } \\
\text { A. et al., } 2006 \text { [26] }\end{array}$ \\
\hline & & ${ }^{206} \mathrm{~Pb} /{ }^{207} \mathrm{~Pb}$ ratio & $\begin{array}{l}\text { Throughout Scotland } \\
\text { (United Kingdom) }\end{array}$ & $\begin{array}{l}\text { Patrick G.J. and } \\
\text { Farmer J.G., } 2007 \\
\text { [69] }\end{array}$ \\
\hline & & PAHs & Palermo area (Sicily, Italy) & $\begin{array}{l}\text { Orecchio S. et al., } \\
2008 \text { [74]. }\end{array}$ \\
\hline & & $\begin{array}{c}\mathrm{pH}\left(\mathrm{SO}_{2}\right) \\
\mathrm{Cd}, \mathrm{Cu}, \mathrm{Pb}, \mathrm{Zn}\end{array}$ & Niepołomice Forest (Poland) & Chrzan A., 2015 [32] \\
\hline & & $\begin{array}{c}\mathrm{pH}\left(\mathrm{SO}_{2}\right) \\
\mathrm{Ca}, \mathrm{Cd}, \mathrm{Cu}, \mathrm{Fe}, \mathrm{K}, \mathrm{Mg}, \mathrm{Mn}, \mathrm{Na}, \mathrm{Ni} \\
\mathrm{Pb}, \mathrm{Zn}\end{array}$ & Rîga (Latvia) & $\begin{array}{l}\text { Čekstere G. et al., } \\
2015 \text { [57] }\end{array}$ \\
\hline & $\begin{array}{c}\text { Pinus } \\
\text { massoniana }\end{array}$ & $\mathrm{Cd}, \mathrm{Co}, \mathrm{Cr}, \mathrm{Cu}, \mathrm{Fe}, \mathrm{Mn}, \mathrm{Ni}, \mathrm{Pb}, \mathrm{Zn}$ & Qujiang, Dinghushan (China) & $\begin{array}{l}\text { Kuang Y.W. et al., } \\
2007[2]\end{array}$ \\
\hline & \multirow{3}{*}{ Pinus nigra } & $\mathrm{pH}\left(\mathrm{SO}_{2}\right)$, electrolytic conductivity & Navarra (Spain) & $\begin{array}{l}\text { Santamaria J.M. and } \\
\text { Martin A., } 1997 \text { [31] }\end{array}$ \\
\hline & & $\mathrm{Cr}, \mathrm{Cu}, \mathrm{Fe}, \mathrm{Pb}$ & $\begin{array}{c}\text { Salzburg (Austria), } \\
\text { Belgrade (Serbia), } \\
\text { Thessaloniki (Greece) }\end{array}$ & $\begin{array}{l}\text { Sawidis T. et al., } \\
\quad 2011[4]\end{array}$ \\
\hline & & $\mathrm{Hg}$ & Mt. Amiata, Toscany (Italy) & $\begin{array}{l}\text { Chiarantini L. et al., } \\
2016 \text { [41] }\end{array}$ \\
\hline & Pinus pinaster & $\begin{array}{c}\mathrm{pH}\left(\mathrm{SO}_{2}\right) \\
\mathrm{Ca}, \mathrm{Cd}, \mathrm{Cu}, \mathrm{K}, \mathrm{Mg}, \mathrm{Na}, \mathrm{Ni}, \mathrm{Pb}, \mathrm{Zn}\end{array}$ & Madrid (Spain) & $\begin{array}{l}\text { Cutillas-Barreiro L. } \\
\text { et al., } 2014 \text { [55] }\end{array}$ \\
\hline & \multirow{2}{*}{ Pinus pinea } & PAHs & $\begin{array}{l}\text { Iskenderun, Hatay Province } \\
\text { (Turkey) }\end{array}$ & $\begin{array}{c}\text { Falay E.O. et al., } \\
2013 \text { [76] }\end{array}$ \\
\hline & & $\begin{array}{l}48 \text { elements including lanthanides (La, } \\
\text { Ce, Pr, Nd, Sm, Gd, Dy, Er, Yb) }\end{array}$ & $\begin{array}{c}\text { Aliağa, Izmir Province } \\
\text { (Turkey) }\end{array}$ & $\begin{array}{l}\text { Odabasi M. et al., } \\
2016[11]\end{array}$ \\
\hline & Pinus resinosa & $\begin{array}{l}\text { PAHs, alkylated-PAHs, nitro and oxy- } \\
\text { PAHs, PBDEs and novel brominated } \\
\text { flame retardants (novel BFRs) }\end{array}$ & Toronto, Ontario (Canada) & $\begin{array}{l}\text { Rauert C. and Harner } \\
\text { T., } 2016[81]\end{array}$ \\
\hline \multirow{2}{*}{ Platanus } & $\begin{array}{l}\text { Platanus } \\
\text { hybrida }\end{array}$ & $\begin{array}{c}\mathrm{Al}, \mathrm{As}, \mathrm{Ba}, \mathrm{Ce}, \mathrm{Co}, \mathrm{Cr}, \mathrm{Cs}, \mathrm{Cu}, \mathrm{Fe}, \mathrm{Hf} \\
\mathrm{Hg}, \mathrm{K}, \mathrm{Mg}, \mathrm{Mn}, \mathrm{Rb}, \mathrm{Sb}, \mathrm{Sc}, \mathrm{Se}, \mathrm{Sm}, \\
\text { Ta, Th, Ti, U, V, Zn }\end{array}$ & Northern Portugal & $\begin{array}{l}\text { Pacheco A.M.G et al., } \\
2008 \text { [67] }\end{array}$ \\
\hline & $\begin{array}{l}\text { Platanus } \\
\text { orientalis }\end{array}$ & $\mathrm{Cr}, \mathrm{Cu}, \mathrm{Fe}, \mathrm{Pb}$ & $\begin{array}{l}\text { Salzburg (Austria), } \\
\text { Belgrade (Serbia), } \\
\text { Thessaloniki (Greece) }\end{array}$ & $\begin{array}{l}\text { Sawidis T. et al., } \\
\quad 2011[4]\end{array}$ \\
\hline \multirow{6}{*}{ Populus } & & $\mathrm{pH}\left(\mathrm{SO}_{2}\right)$, buffer capacity & Stockholm (Sweden) & Skye E. 1968 [21] \\
\hline & Populus sp. & $\begin{array}{c}\mathrm{pH}\left(\mathrm{SO}_{2}\right) \\
\mathrm{As}, \mathrm{Br}, \mathrm{Ca}, \mathrm{Cd}, \mathrm{Ce}, \mathrm{Co}, \mathrm{Cr}, \mathrm{Cs}, \mathrm{Fe}, \mathrm{Hg}, \\
\mathrm{K}, \mathrm{La}, \mathrm{Na}, \mathrm{Ni}, \mathrm{Pb}, \mathrm{Rb}, \mathrm{Sb}, \mathrm{Sc}, \mathrm{Se}, \mathrm{Sm}, \\
\mathrm{Th}, \mathrm{Zn} ; \\
\mathrm{NH}_{4}^{+}, \mathrm{NO}_{3}^{+}, \mathrm{SO}_{4}^{+2}\end{array}$ & \multirow[t]{2}{*}{ Throughout the Netherlands } & $\begin{array}{l}\text { Wolterbeek H. Th. et } \\
\text { al., } 1996 \text { [62] }\end{array}$ \\
\hline & $\begin{array}{l}\text { Populus } x \\
\text { canadensis }\end{array}$ & $\begin{array}{c}\mathrm{pH}\left(\mathrm{SO}_{2}\right) \\
\mathrm{As}, \mathrm{Br}, \mathrm{Ca}, \mathrm{Cd}, \mathrm{Ce}, \mathrm{Co}, \mathrm{Cr}, \mathrm{Cs}, \mathrm{Fe}, \mathrm{Hg}, \\
\text { K, } \mathrm{La}, \mathrm{Na}, \mathrm{Ni}, \mathrm{Sb}, \mathrm{Sc}, \mathrm{Se}, \mathrm{Sm}, \mathrm{Th}, \mathrm{Zn} \\
\mathrm{NH}_{4}^{+}, \mathrm{NO}_{3}^{+}, \mathrm{SO}_{4}^{+2}\end{array}$ & & $\begin{array}{l}\text { Van Dobben H.F. et } \\
\text { al., } 2001[24]\end{array}$ \\
\hline & \multirow{2}{*}{ Populus nigra } & $\begin{array}{c}\text { As, } \mathrm{Au}, \mathrm{Ce}, \mathrm{Co}, \mathrm{Cr}, \mathrm{Cu}, \mathrm{La}, \mathrm{Mn}, \mathrm{Mo} \\
\text { Ni, Sb, Sm, Ti, Th, U, V, W }\end{array}$ & $\begin{array}{c}\text { Kiev } \\
\text { (Ukraine) }\end{array}$ & $\begin{array}{l}\text { Berlizov A.N. et al., } \\
2007 \text { [39] }\end{array}$ \\
\hline & & $\mathrm{Cd}, \mathrm{Cu}, \mathrm{Ni}, \mathrm{Pb}, \mathrm{Zn}$ & $\begin{array}{c}\text { Navodari } \\
\text { (Romania) } \\
\end{array}$ & $\begin{array}{c}\text { Barbeş L. et al., } 2014 \\
{[54]}\end{array}$ \\
\hline & $\begin{array}{l}\text { Populus } \\
\text { tremula }\end{array}$ & $\begin{array}{l}\mathrm{Cd}, \mathrm{Cu}, \mathrm{Hg}, \mathrm{Pb}, \mathrm{S}, \mathrm{Zn} \\
\text { PAHs, phenols }\end{array}$ & $\begin{array}{l}\text { Świętokrzyskie Mountains } \\
\text { (Poland) }\end{array}$ & $\begin{array}{l}\text { Migaszewski Z. et al., } \\
2002[25]\end{array}$ \\
\hline Prunus & Prunus serrula & $\begin{array}{c}\mathrm{Ag}, \mathrm{Al}, \mathrm{As}, \mathrm{Ba}, \mathrm{Cd}, \mathrm{Co}, \mathrm{Cr}, \mathrm{Cu}, \mathrm{Fe}, \mathrm{Mn} \\
\text { Ni, Pb, Sb, Se, Sn, Ti, V, Zn }\end{array}$ & Sheffield (United Kingdom) & $\begin{array}{l}\text { Schelle E. et al., } 2008 \\
\text { [8] }\end{array}$ \\
\hline
\end{tabular}


Table 1. Continued.

\begin{tabular}{|c|c|c|c|c|}
\hline \multirow{16}{*}{ Quercus } & Quercus sp. & $\begin{array}{c}\mathrm{pH}\left(\mathrm{SO}_{2}\right) \\
\mathrm{As}, \mathrm{Br}, \mathrm{Ca}, \mathrm{Cd}, \mathrm{Ce}, \mathrm{Co}, \mathrm{Cr}, \mathrm{Cs}, \mathrm{Fe}, \mathrm{Hg}, \\
\mathrm{K}, \mathrm{La}, \mathrm{Na}, \mathrm{Ni}, \mathrm{Pb}, \mathrm{Rb}, \mathrm{Sb}, \mathrm{Sc}, \mathrm{Se}, \mathrm{Sm} \\
\mathrm{Th}, \mathrm{Zn} ; \\
\mathrm{NH}_{4}^{+}, \mathrm{NO}_{3}^{+}, \mathrm{SO}_{4}^{+2}\end{array}$ & throughout Netherlands & $\begin{array}{l}\text { Wolterbeek H. Th. et } \\
\text { al., } 1996 \text { [62] }\end{array}$ \\
\hline & $\begin{array}{l}\text { Quercus } \\
\text { crispula }\end{array}$ & As & $\begin{array}{l}\text { Ashio, Tochigi prefecture } \\
\text { (Japan) }\end{array}$ & $\begin{array}{l}\text { Bellis D.J. et al., } \\
2003 \text { [61] }\end{array}$ \\
\hline & $\begin{array}{l}\text { Quercus } \\
\text { faginea }\end{array}$ & $\mathrm{pH}\left(\mathrm{SO}_{2}\right)$, electrolytic conductivity & Navarra (Spain) & $\begin{array}{l}\text { Santamaria J.M. and } \\
\text { Martin A. } 1997 \text { [31] }\end{array}$ \\
\hline & \multirow{3}{*}{ Quercus ilex } & $\mathrm{pH}\left(\mathrm{SO}_{2}\right)$, electrolytic conductivity & Navarra (Spain) & $\begin{array}{l}\text { Santamaria J.M. and } \\
\text { Martin A. } 1997 \text { [31] }\end{array}$ \\
\hline & & As, $\mathrm{Cd}, \mathrm{Co}, \mathrm{Cu}, \mathrm{Fe}, \mathrm{Mn}, \mathrm{Ni}, \mathrm{Pb}, \mathrm{V}, \mathrm{Zn}$ & Genova, Liguria (Italy) & $\begin{array}{c}\text { Drava G. et al., } 2016 \\
{[9]}\end{array}$ \\
\hline & & As, $\mathrm{Cd}, \mathrm{Co}, \mathrm{Cu}, \mathrm{Fe}, \mathrm{Mn}, \mathrm{Ni}, \mathrm{Pb}, \mathrm{V}, \mathrm{Zn}$ & $\begin{array}{l}\text { Genova, Rapallo, Liguria } \\
\text { (Italy) }\end{array}$ & $\begin{array}{l}\text { Minganti V. et al., } \\
2016 \text { [35] }\end{array}$ \\
\hline & $\begin{array}{l}\text { Quercus } \\
\text { petraea }\end{array}$ & $\begin{array}{c}\text { Al, As, Ce, Co, Cr, Cs, Fe, Hf, } \\
\mathrm{Hg}, \mathrm{La}, \mathrm{Mn}, \mathrm{Na}, \mathrm{Ni}, \mathrm{Rb}, \mathrm{S}, \mathrm{Sb}, \mathrm{Sc}, \mathrm{Se} \\
\text { Th, Ti, U, V, W, Zn }\end{array}$ & $\begin{array}{l}\text { Throughout the Czech Re- } \\
\text { public }\end{array}$ & $\begin{array}{l}\text { Böhm P. et al., } 1998 \\
\text { [1] }\end{array}$ \\
\hline & $\begin{array}{l}\text { Quercus } \\
\text { pubescens }\end{array}$ & $\begin{array}{c}\text { As, } \mathrm{Cd}, \mathrm{Co}, \mathrm{Cr}, \mathrm{Cu}, \mathrm{Fe}, \mathrm{Hg}, \mathrm{Mn}, \mathrm{Ni}, \mathrm{P}, \\
\mathrm{Pb}, \mathrm{Sn}, \mathrm{Zn} ; \\
{ }^{15} \mathrm{~N},{ }^{18} \mathrm{O},{ }^{13} \mathrm{C} ; \\
\text { PCDDs, PCDFs }\end{array}$ & Venafro Plain, Molise (Italy) & $\begin{array}{l}\text { Cocozza C. et al., } \\
2016[28]\end{array}$ \\
\hline & \multirow{8}{*}{ Quercus robur } & \multirow{3}{*}{$\mathrm{pH}\left(\mathrm{SO}_{2}\right)$, buffer capacity } & Stockholm (Sweden) & Skye E. 1968 [21] \\
\hline & & & $\begin{array}{c}\text { Białowieża National Park, } \\
\text { Niepołomice Forest (Poland) }\end{array}$ & $\begin{array}{c}\text { Grodzińska K. 1977, } \\
1979[29,30] \\
\end{array}$ \\
\hline & & & Navarra (Spain) & $\begin{array}{l}\text { Santamaria J.M. and } \\
\text { Martin A. } 1997 \text { [31] }\end{array}$ \\
\hline & & $\begin{array}{c}\text { Al, As, Ce, Co, Cr, Cs, Fe, Hf, } \\
\mathrm{Hg}, \mathrm{La}, \mathrm{Mn}, \mathrm{Na}, \mathrm{Ni}, \mathrm{Rb}, \mathrm{S}, \mathrm{Sb}, \mathrm{Sc}, \mathrm{Se} \\
\text { Th, Ti, U, V, W, Zn }\end{array}$ & Western Czech Republic & $\begin{array}{l}\text { Böhm P. et al., } 1998 \\
\text { [1] }\end{array}$ \\
\hline & & $\begin{array}{c}\mathrm{pH}\left(\mathrm{SO}_{2}\right) \\
\mathrm{As}, \mathrm{Br}, \mathrm{Ca}, \mathrm{Cd}, \mathrm{Ce}, \mathrm{Co}, \mathrm{Cr}, \mathrm{Cs}, \mathrm{Fe}, \mathrm{Hg}, \\
\mathrm{K}, \mathrm{La}, \mathrm{Na}, \mathrm{Ni}, \mathrm{Sb}, \mathrm{Sc}, \mathrm{Se}, \mathrm{Sm}, \mathrm{Th}, \mathrm{Zn} \\
\mathrm{NH}_{4}^{+}, \mathrm{NO}_{3}^{+}, \mathrm{SO}_{4}^{+2}\end{array}$ & Throughout the Netherlands & $\begin{array}{l}\text { Van Dobben H.F. et } \\
\text { al., } 2001[24]\end{array}$ \\
\hline & & $\begin{array}{l}\mathrm{Cd}, \mathrm{Cu}, \mathrm{Hg}, \mathrm{Pb}, \mathrm{S}, \mathrm{Zn} \\
\text { PAHs, phenols }\end{array}$ & $\begin{array}{c}\text { Świętokrzyskie Mountains } \\
\text { (Poland) }\end{array}$ & $\begin{array}{l}\text { Migaszewski Z. et al., } \\
2002[25]\end{array}$ \\
\hline & & $\begin{array}{c}\mathrm{Ag}, \mathrm{Al}, \mathrm{As}, \mathrm{Ba}, \mathrm{Cd}, \mathrm{Co}, \mathrm{Cr}, \mathrm{Cu}, \mathrm{Fe}, \mathrm{Mn} \\
\mathrm{Ni}, \mathrm{Pb}, \mathrm{Sb}, \mathrm{Se}, \mathrm{Sn}, \mathrm{Ti}, \mathrm{V}, \mathrm{Zn}\end{array}$ & Sheffield (United Kingdom) & $\begin{array}{c}\text { Schelle E. et al., } 2008 \\
\text { [7] }\end{array}$ \\
\hline & & ${ }^{137} \mathrm{Cs}$ & $\begin{array}{c}\text { Northwest, southwest, and } \\
\text { central Romania }\end{array}$ & $\begin{array}{c}\text { Cosma C. et al., } 2016 \\
{[70]} \\
\end{array}$ \\
\hline \multirow[t]{2}{*}{ Robinia } & \multirow{2}{*}{$\begin{array}{c}\text { Robinia } \\
\text { pseudoacaccia. }\end{array}$} & $\mathrm{Cd}, \mathrm{Co}, \mathrm{Cr}, \mathrm{Cu}, \mathrm{Fe}, \mathrm{Mn}, \mathrm{Ni}, \mathrm{Pb}, \mathrm{Zn}$ & $\begin{array}{l}\text { Oleśnica, Lower Silesia } \\
\text { Province } \\
\text { (Poland) } \\
\end{array}$ & $\begin{array}{l}\text { Samecka-Cymerman } \\
\text { A. et al., } 2009 \text { [3] }\end{array}$ \\
\hline & & $\mathrm{pH}\left(\mathrm{SO}_{2}\right)$ & $\begin{array}{l}\text { cities of Upper Silesian } \\
\text { Industrial Region (Poland) }\end{array}$ & $\begin{array}{l}\text { Steindor K. et al., } \\
2010[45]\end{array}$ \\
\hline \multirow{3}{*}{ Salix } & \multirow[b]{2}{*}{ Salix sp. } & $\mathrm{pH}\left(\mathrm{SO}_{2}\right)$, buffer capacity & Stockholm (Sweden) & Skye E. 1968 [21] \\
\hline & & $\begin{array}{c}\mathrm{pH}\left(\mathrm{SO}_{2}\right) ; \\
\mathrm{As}, \mathrm{Br}, \mathrm{Ca}, \mathrm{Cd}, \mathrm{Ce}, \mathrm{Co}, \mathrm{Cr}, \mathrm{Cs}, \mathrm{Fe}, \mathrm{Hg} \\
\mathrm{K}, \mathrm{La}, \mathrm{Na}, \mathrm{Ni}, \mathrm{Pb}, \mathrm{Rb}, \mathrm{Sb}, \mathrm{Sc}, \mathrm{Se}, \mathrm{Sm}, \\
\mathrm{Th}, \mathrm{Zn} ; \\
\mathrm{NH}_{4}^{+}, \mathrm{NO}_{3}^{+}, \mathrm{SO}_{4}^{+2}\end{array}$ & Throughout the Netherlands & $\begin{array}{l}\text { Wolterbeek H. Th. et } \\
\text { al., } 1996 \text { [62] }\end{array}$ \\
\hline & Salix alba & $\begin{array}{c}\mathrm{pH}\left(\mathrm{SO}_{2}\right) \\
\mathrm{As}, \mathrm{Br}, \mathrm{Ca}, \mathrm{Cd}, \mathrm{Ce}, \mathrm{Co}, \mathrm{Cr}, \mathrm{Cs}, \mathrm{Fe}, \mathrm{Hg} \\
\mathrm{K}, \mathrm{La}, \mathrm{Na}, \mathrm{Ni}, \mathrm{Sb}, \mathrm{Sc}, \mathrm{Se}, \mathrm{Sm}, \mathrm{Th}, \mathrm{Zn} \\
\mathrm{NH}_{4}^{+}, \mathrm{NO}_{3}^{+}, \mathrm{SO}_{4}^{+2}\end{array}$ & Throughout the Netherlands & $\begin{array}{l}\text { Van Dobben H.F. et } \\
\text { al., } 2001[24]\end{array}$ \\
\hline
\end{tabular}


Table 1. Continued.

\begin{tabular}{|c|c|c|c|c|}
\hline \multirow{2}{*}{ Taxus } & \multirow{2}{*}{ Taxus baccata } & $\mathrm{pH}\left(\mathrm{SO}_{2}\right)$ & $\begin{array}{l}\text { Cities of the Upper Silesian } \\
\text { Industrial Region (Poland) }\end{array}$ & $\begin{array}{l}\text { Steindor K. et al., } \\
2010[45]\end{array}$ \\
\hline & & $\begin{array}{c}\mathrm{Ca}, \mathrm{Cd}, \mathrm{Co}, \mathrm{Cr}, \mathrm{Cu}, \mathrm{Fe}, \mathrm{K}, \mathrm{Mg}, \mathrm{Mn}, \mathrm{N}, \\
\mathrm{Ni}, \mathrm{P}, \mathrm{Pb}, \mathrm{Zn}\end{array}$ & $\begin{array}{l}\text { Wrocław, Lower Silesia } \\
\text { Province (Poland) }\end{array}$ & $\begin{array}{l}\text { Samecka-Cymerman } \\
\text { A. et al., } 2011 \text { [51] }\end{array}$ \\
\hline \multirow[t]{2}{*}{ Terminalia } & \multirow{2}{*}{$\begin{array}{l}\text { Terminalia } \\
\text { catappa }\end{array}$} & $\mathrm{Cu}, \mathrm{Fe}, \mathrm{Mn}, \mathrm{Ni}, \mathrm{Pb}, \mathrm{Zn}$ & $\begin{array}{l}\text { Ogbomoso } \\
\text { (Nigeria) }\end{array}$ & $\begin{array}{c}\text { Olajire A.A. and } \\
\text { Ayodele E.T. } 2003 \\
\text { [49]. }\end{array}$ \\
\hline & & $\mathrm{Ba}, \mathrm{Al}, \mathrm{Fe}, \mathrm{K}, \mathrm{Sr}, \mathrm{Zn}$ & $\begin{array}{c}\text { Paranaguá Paraná state } \\
\text { (Brazil) }\end{array}$ & $\begin{array}{l}\text { Gurgatz M. et al., } \\
2016[10]\end{array}$ \\
\hline \multirow{7}{*}{ Tilia } & \multirow{4}{*}{ Tilia $\mathrm{sp}$. } & $\mathrm{pH}\left(\mathrm{SO}_{2}\right)$, buffer capacity & Stockholm (Sweden) & Skye E. 1968 [21] \\
\hline & & $\begin{array}{c}\mathrm{As}, \mathrm{Cd}, \mathrm{Co}, \mathrm{Cr}, \mathrm{Cu}, \mathrm{Fe}, \mathrm{Mn}, \mathrm{Mo}, \mathrm{Ni} \\
\mathrm{Pb}, \mathrm{Sb}, \mathrm{Sn}, \mathrm{V}, \mathrm{Zn} \\
\mathrm{PCBs}, \mathrm{PCDD} / \mathrm{Fs}\end{array}$ & \multirow[b]{2}{*}{$\begin{array}{l}\text { Strasburg (France) } \\
\text { Kehl (Germany) }\end{array}$} & $\begin{array}{l}\text { Guéguen F. et al., } \\
2011 \text { [12] }\end{array}$ \\
\hline & & $\begin{array}{l}\mathrm{Al}, \mathrm{As}, \mathrm{Ba}, \mathrm{Ca}, \mathrm{Cd}, \mathrm{Ce}, \mathrm{Co}, \mathrm{Cr}, \mathrm{Cs}, \mathrm{Cu} \\
\mathrm{Dy}, \mathrm{Er}, \mathrm{Eu}, \mathrm{Fe}, \mathrm{Gd}, \mathrm{Ho}, \mathrm{K}, \mathrm{La}, \mathrm{Lu}, \mathrm{Mg} \text {, } \\
\mathrm{Mn}, \mathrm{Mo}, \mathrm{Na}, \mathrm{Nd}, \mathrm{Ni}, \mathrm{P}, \mathrm{Pb}, \mathrm{Pr}, \mathrm{Rb}, \mathrm{Sb} \\
\mathrm{Si}, \mathrm{Sm}, \mathrm{Sn}, \mathrm{Sr}, \mathrm{Tb}, \mathrm{Th}, \mathrm{Ti}, \mathrm{Tm}, \mathrm{U}, \mathrm{V}, \mathrm{Y} \\
\mathrm{Yb}, \mathrm{Zn}, \mathrm{Zr} \\
{ }^{87} \mathrm{Sr} /{ }^{86} \mathrm{Sr},{ }^{143} \mathrm{Nd} /{ }^{144} \mathrm{Nd},{ }^{206} \mathrm{~Pb} /{ }^{207} \mathrm{~Pb} \text { ratios }\end{array}$ & & $\begin{array}{l}\text { Guéguen F. et al., } \\
\text { 2012, } 2012[62,63]\end{array}$ \\
\hline & & $\mathrm{Cd}, \mathrm{Co}, \mathrm{Cr}, \mathrm{Cu}, \mathrm{Fe}, \mathrm{Hg}, \mathrm{Mn}, \mathrm{Ni}, \mathrm{Pb}, \mathrm{Zn}$ & $\begin{array}{c}\text { Banja Luka } \\
\text { (Bosnia and Herzegovina) }\end{array}$ & $\begin{array}{l}\text { Škarbić B. et al., } \\
2012[40]\end{array}$ \\
\hline & \multirow{2}{*}{ Tilia cordata } & $\mathrm{pH}\left(\mathrm{SO}_{2}\right), \mathrm{Ca}, \mathrm{K}, \mathrm{Na}$ & $\begin{array}{c}\text { Frankfurt am Main, Hesse } \\
\text { (Germany) }\end{array}$ & $\begin{array}{l}\text { Lötschert W. and } \\
\text { Köhm H.J. } 1977 \text { [46] }\end{array}$ \\
\hline & & $\mathrm{pH}\left(\mathrm{SO}_{2}\right)$, buffer capacity & $\begin{array}{l}\text { Białowieża National Park, } \\
\text { Niepołomice Forest (Poland) }\end{array}$ & $\begin{array}{c}\text { Grodzińska K. 1977, } \\
1979[29,30]\end{array}$ \\
\hline & $\begin{array}{l}\text { Tilia } \\
\text { platyphyllos }\end{array}$ & $\mathrm{pH}\left(\mathrm{SO}_{2}\right), \mathrm{Ca}, \mathrm{K}, \mathrm{Na}$ & Frankfurt (Germany) & $\begin{array}{l}\text { Lötschert W. and } \\
\text { Köhm H.J. } 1977 \text { [46] }\end{array}$ \\
\hline Tipuana & Tipuana tipu & $\begin{array}{c}\mathrm{Al}, \mathrm{Ba}, \mathrm{Ca}, \mathrm{Cl}, \mathrm{Cu}, \mathrm{Fe}, \mathrm{K}, \mathrm{Mg}, \mathrm{Mn}, \mathrm{Na} \\
\text { P, Rb, S, Sr, Zn }\end{array}$ & $\begin{array}{c}\text { São } \\
\text { Paulo (Brazil) }\end{array}$ & $\begin{array}{l}\text { Moreira T.C.L. et al., } \\
2016[60]\end{array}$ \\
\hline \multirow{4}{*}{ Ulmus } & \multirow[b]{2}{*}{ Ulmus sp. } & $\mathrm{pH}\left(\mathrm{SO}_{2}\right)$, buffer capacity & Stockholm (Sweden) & Skye E. 1968 [21] \\
\hline & & $\begin{array}{c}\mathrm{pH}\left(\mathrm{SO}_{2}\right) ; \\
\mathrm{As}, \mathrm{Br}, \mathrm{Ca}, \mathrm{Cd}, \mathrm{Ce}, \mathrm{Co}, \mathrm{Cr}, \mathrm{Cs}, \mathrm{Fe}, \mathrm{Hg}, \\
\mathrm{K}, \mathrm{La}, \mathrm{Na}, \mathrm{Ni}, \mathrm{Pb}, \mathrm{Rb}, \mathrm{Sb}, \mathrm{Sc}, \mathrm{Se}, \mathrm{Sm} \\
\mathrm{Th}, \mathrm{Zn} ; \\
\mathrm{NH}_{4}^{+}, \mathrm{NO}_{3}^{+}, \mathrm{SO}_{4}^{+2}\end{array}$ & Throughout the Netherlands & $\begin{array}{l}\text { Wolterbeek H. Th. } \\
\text { et al., } 1996 \text { [62] }\end{array}$ \\
\hline & $\begin{array}{l}\text { Ulmus } x \\
\text { hollandica }\end{array}$ & $\begin{array}{c}\mathrm{pH}\left(\mathrm{SO}_{2}\right) \\
\mathrm{As}, \mathrm{Br}, \mathrm{Ca}, \mathrm{Cd}, \mathrm{Ce}, \mathrm{Co}, \mathrm{Cr}, \mathrm{Cs}, \mathrm{Fe}, \mathrm{Hg} \\
\mathrm{K}, \mathrm{La}, \mathrm{Na}, \mathrm{Ni}, \mathrm{Sb}, \mathrm{Sc}, \mathrm{Se}, \mathrm{Sm}, \mathrm{Th}, \mathrm{Zn} \\
\mathrm{NH}_{4}^{+}, \mathrm{NO}_{3}^{+}, \mathrm{SO}_{4}^{+2}\end{array}$ & Throughout the Netherlands & $\begin{array}{l}\text { Van Dobben H.F. } \\
\text { et al., } 2001[24]\end{array}$ \\
\hline & Ulmus rubra & $\mathrm{U}$ & southwest Ohio (USA) & $\begin{array}{l}\text { Conte E.R. et al., } \\
2016 \text { [14] }\end{array}$ \\
\hline
\end{tabular}

${ }^{*} \mathrm{pH}\left(\mathrm{SO}_{2}\right)$ - bark acidity test for the sulphur contained.

wherein many of these compounds are deliberately introduced into the environment as cheap and efficient pesticides [80]. Determining organohalogen compounds (OHCs) like organochlorine pesticides (OCPs), dechlorane plus (DP), and novel brominated flame retardants (NBFRs) in tree bark has recently been described by Chinese researchers. Material examined from rapidly growing city of Kaiyuan in Yunnan Province [83] and the Yellow River region [85] showed substantial contamination of these compounds in China and promising properties of the bark as a sensitive marker of this ubiquitous organic compound in the environment.

\section{Examples of Air Quality Determination Using the Bark Deriving from Various Species of Trees}

Tree bark is in large part a dead organ. Therefore, it is treated by most authors as a passive indicator of air pollution. It might appear as if the bark of most tree species can act as an air pollution bioindicator which, however, turns out not to be true $[2,20]$. Bark is an organ with its own unique chemical characteristics, such as distinctive acidity depending on the species [21, 29-32, 42, 44]. It is well known that the $\mathrm{pH}$ value influences the absorption 
of elements from soil, and, therefore, bark acidity might interfere with the efficient adsorption of impurities, e.g., heavy metals [33-34]. Therefore, for effective air monitoring a species of known biology should be selected and, where possible, with stable chemical conditions. Table 1 shows selected scientific reports showing the potential use of bark of various trees in the identification of air pollution over the last half century.

Studies of air quality using tree bark both in Poland and in other European countries are especially often conducted using the bark of trees from the genus Pinus, including Scots pine (Pinus sylvestris) [25, 29-32, 38, $44,57,63-64,68-69]$, the needles of which are a well documented bioindicator of air pollution. As a species common to nearly all of north-central Europe, P. sylvestris is a valuable source of information on the spread of atmospheric pollutants, both organic and inorganic. The usefulness of pine bark is, beyond its high porosity, based on a complete lack of metabolic processes in the dead part, where processes of dust accumulation may occur. As a result, Scots pine bark almost perfectly reflects the level of contamination of organic and inorganic substances [6364]. Despite the fact that the Scots pine is a species tolerant to soil type and $\mathrm{pH}$, it prefers acidic soils, which may affect the results. Furthermore, the pine is a coniferous species, which sometimes cannot fully represent the area. Among deciduous species, bark samples from the oak Quercus sp., mostly Q. robur [1, 7, 21, 24-25, 29-31, 70], or linden (Tilia sp.) [12, 21, 29-30, 40, 46, 63] are used equally often to study air quality. Bioindication of air quality in Europe is also often based on the analysis of the bark of the ash, beech, or poplar [12, 21, 24-25, 29-31, 33-34, 38-39, 45, 46, 62]. In northern Europe, trees of the genus Betula are especially popular species whose bark is used to determine air quality $[21,69]$. On the other hand, in the southern part of Europe, the common olive tree is a popular species for testing air quality [65-66].

\section{Conclusions}

Tree bark is a valuable source of information about pollutants in the environment which is confirmed by scientific reports. The use of tree bark in assessing air quality is particularly useful because of:

1. Absorbent properties of the bark, enabling determination of permanent and temporary air pollution of different chemical origins

2. The ability to compare data with the selection of common species in the studied areas and the ability to map the spread of contamination and identify emitters and pollutants

3. Observation of the effect of pollution on the condition of the material (ecotoxicology)

4. Wide availability of the material, ease, and low costs of sampling

Despite the fact that elemental analysis remains the dominant direction of environmental research based on tree bark, far-reaching progress has been made over the last 50 years on approaches to the use of the plant material in monitoring air quality. The most commonly performed physical and chemical tests of air quality relate only to determine the temporary content of impurities in the air. There is no doubt that the inclusion of bioindicative research, including the use of tree bark in standard physicochemical air monitoring would enable holistic analysis of air quality. Such monitoring could provide information on both temporary and periodic air pollution as well as valuable ecotoxicological information.

\section{References}

1. BÖHM P., WOLTERBEEK H., VERBURG T., MUSILEK L. The use of tree bark for environmental pollution monitoring in the Czech Republic. Environ. Pollut. 102 (2), 243, 1998.

2. KUANG Y.W., ZHOU G.Y., WEN D.Z., LIU S.Z. Heavy metals in bark of Pinus massoniana (Lamb.) as an indicator of atmospheric deposition near a smeltery at Qujiang, China. Environ. Environ Sci. Pollut. R. 14 (4), 270, 2007.

3. SAMECKA-CYMERMAN A., STANKIEWICZ A., KOLON K., KEMPERS A.J. Self-organizing feature map (neural networks) as a tool to select the best indicator of road traffic pollution (soil, leaves or bark of Robinia pseudoacacia L.). Environ. Pollut. 157 (7), 2061, 2009.

4. SAWIDIS T., BREUSTE J., MITROVIC M., PAVLOVIC P., TSIGARIDAS K. Trees as bioindicator of heavy metal pollution in three European cities. Environ. Pollut. 159 (12), 3560, 2011.

5. COCOZZA C., RAVERA S., CHERUBINI P., LOMBARDI F., MARCHETTI M., TOGNETTI R. Integrated biomonitoring of airborne pollutants over space and time using tree rings, bark, leaves and epiphytic lichens. Urban For. Urban Gree. 17, 177, 2016.

6. KALINOWSKA A. Acid rains, pollution knows no boundaries. Ecology- the choice of the future. Editions Spotkania wyd. II; 132, Warszawa, 1994. [In Polish].

7. HARRISON R.M., YIN J. Particulate matter in the atmosphere: which particle properties are important for its effects on health? Sci. Total Environ. 249 (1) 85, 2000.

8. SCHELLE E., RAWLINS B.G., LARK R.M., WEBSTER R., STATON I., MCLEOD C.W. Mapping aerial metal deposition in metropolitan areas from tree bark: a case study in Sheffield, England. Environ. Pollut. 155 (1), 164, 2008.

9. DRAVA G., BRIGNOLE D., GIORDANI P., MINGANTI V. Urban and industrial contribution to trace elements in the atmosphere as measured in Holm oak bark. Atmos. Environ. 144, 370, 2016.

10. GURGATZ B.M., CARVALHO-OLIVEIRA R., DE OLIVEIRA D.C., JOUCOSKI E., ANTONIACONI G., DO NASCIMENTO SALDIVA P.H., REIS R.A. Atmospheric metal pollutants and environmental injustice: A methodological approach to environmental risk analysis using fuzzy logic and tree bark. Ecol. Indic. 71, 428, 2016.

11. ODABASI M., TOLUNAY D., KARA M., FALAY E.O., TUNA G., ALTIOK H., ELBIR T. Investigation of spatial and historical variations of air pollution around an industrial region using trace and macro elements in tree components. Sci. Total Environ. 550, 1010, 2016.

12. GUÉGUEN F., STILLE P., MILLET M. Air quality assessment by tree bark biomonitoring in urban, industrial and rural environments of the Rhine Valley: PCDD/Fs, PCBs and trace metal evidence. Chemosphere. 85 (2), 195, 2011. 
13. OLATUNJI O.S., OSIBANJO O., FATOKI O.S., XIMBA B.J., OPEOLU B.O. Selected heavy metals as indices of atmospheric pollution in African locust bean (Parkia biglobosa) tree barks. Am. J. Environ. Sci. 12 (1), 48, 2016.

14. CONTE E.R., WIDOM E., KUENTZ D. Characterization and transport modeling of uranium particle from Fernald area tree bark. J. Radioanal. Nucl. Chem. 307 (3), 1675, 2016.

15. DOCKERY D.W., POPE C.A. Acute respiratory effects of particulate air pollution. Annu. Rev. Publ. Health. 15 (1), 107, 1994.

16. SEATON A., GODDEN D., MACNEE W., DONALDSON K. Particulate air pollution and acute health effects. Lancet. 345 (8943), 176, 1995.

17. WHO (WORLD HEALTH ORGANIZATION). Review of Evidence on Health Aspects of Air Pollution - REVIHAAP Project. Technical Report. World Health Organization, Regional Office for Europe, Copenhagen, Denmark, 2013.

18. KABATA-PENDIAS A. Soil - plant transfer of trace elements- an environmental issue. Geoderma. 122 (2), 143, 2004.

19. EL- HASAN T., AL- OMARI H., JIRIES A., AL- NASIR F., Cypress tree (Cupressus semervirens L.) bark as an indicator for heavy metal pollution in the atmosphere of Amman City, Jordan. Environ. Int. 28 (6), 513, 2002.

20. ERNST W.H.O. The use of higher plants as bioindicators. Trace metals and other contaminants in the environment; Markert B.A., Breure A.M., Zechmeister H.G. Eds., Elsevier, Vol. 6: Bioindicators and biomonitors, Principles, Concepts and Applications; 423, Netherlands, 2003.

21. SKYE E. Lichens as biological indicators of air pollution. Annu. Rev. Phytopathol. 17 (1), 325, 1979.

22. DE WIT T. Lichens as indicators for air quality. Environ. Monit. Assess. 3 (3-4), 273, 1983.

23. GRODZIŃSKA K., SZAREK-ŁUKASZEWSKA G. Response of mosses to the heavy metal deposition in Polandan overview. Environ. Pollut. 114 (3), 443, 2001.

24. VAN DOBBEN H.F., WOLTERBEEK H.T., WAMELINK G.W.W., TER BRAAK C.J.F. Relationship between epiphytic lichens, trace elements and gaseous atmospheric pollutants. Environ. Pollut. 112 (2), 163, 2001.

25. MIGASZEWSKI Z.M., GAŁUSZKA A., PASŁAWSKI P. Polynuclear aromatic hydrocarbons, phenols, and trace metals in selected soil profiles and plant bioindicators in the Holy Cross Mountains, South-Central Poland. Environ. Int. 28 (4), 303, 2002.

26. SAMECKA-CYMERMAN A., KOSIOR G., KEMPERS A.J. Comparison of the moss Pleurozium schreberi with needles and bark of Pinus sylvestris as biomonitors of pollution by industry in Stalowa Wola (southeast Poland). Ecotox. Environ. Safe. 65 (1), 108, 2006.

27. HISSLER C., STILLE P., KREIN A., GEAGEA M.L., PERRONE T., PROBST J.L., HOFFMANN L. Identifying the origins of local atmospheric deposition in the steel industry basin of Luxembourg using the chemical and isotopic composition of the lichen Xanthoria parietina. Sci. Total Environ. 405 (1), 338, 2008

28. COCOZZA C., RAVERA S., CHERUBINI P., LOMBARDI F., MARCHETTI M., TOGNETTI R. Integrated biomonitoring of airborne pollutants over space and time using tree rings, bark, leaves and epiphytic lichens. Urban For. Urban Gree. 17, 177, 2016.

29. GRODZIŃSKA K. Acidity of tree bark as a bioindicator of forest pollution in southern Poland. Water Air Soil Poll. 8 (1), 3, 1977.
30. GRODZIŃSKA K. Tree bark- sensitive biotest for environment acidification. Environ. Int. 2 (3), 173, 1979.

31. SANTAMARIA J.M., MARTIN A. Tree bark as a bioindicator of air pollution in Navarra, Spain. Water Air Soil Poll. 98 (3-4), 381, 1997.

32. CHRZAN A. Necrotic bark of common pine (Pinus sylvestris L.) as a bioindicator of environmental quality. Environ. Sci. Pollut. R. 22 (2), 1066, 2015.

33. CATINON M., AYRAULT S., CLOCCHIATTI R., BOUDOUMA O., ASTA J., TISSUT M., RAVANEL P. The anthropogenic atmospheric elements fraction: a new interpretation of elemental deposits on tree barks. Atmos. Environ. 43 (5), 1124, 2009.

34. CATINON M., AYRAULT S., BOUDOUMA O., ASTA J., TISSUT M., RAVANEL P. Atmospheric element deposit on tree barks: The opposite effects of rain and transpiration. Ecol. Indic. 14 (1), 170, 2012.

35. MINGANTI V., DRAVA G., GIORDANI P., MALASPINA P., MODENESI P. Human contribution to trace elements in urban areas as measured in Holm oak (Quercus ilex L.) bark. Environ. Sci. Pollut. R. 23 (12), 12467, 2016.

36. MARTIN R.E., CRIST J.B. Elements of bark structure and terminology. Wood Fiber Sci. 2, (3), pp. 269-279, 2007.

37. JANTA R., CHANTARA S., INTA A., KAWASHIMA M., SATAKE K. Levels of Road Traffic Heavy Metals in Tree Bark Layers of Cassia fistula Tree. IJESD. 7 (5), 385, 2016.

38. BELLIS D., MA R., BRAMALL N., MCLEOD C. W., CHAPMAN N., SATAKE K. Airborne uranium contamination- as revealed through elemental and isotopic analysis of tree bark. Environ. Pollut. 114 (3), 383, 2001.

39. BERLIZOV A.N., BLUM O.B., FILBY R.H., MALYUK I.A., TRYSHYN V.V. Testing applicability of black poplar (Populus nigra L.) bark to heavy metal air pollution monitoring in urban and industrial regions. Sci. Total Environ. 372 (2), 693, 2007.

40. ŠKRBIĆ B., MILOVAC S., MATAVULJ M. Multielement profiles of soil, road dust, tree bark and wood- rotten fungi collected at various distances from high- frequency road in urban area. Ecol. Indic. 13 (1), 168, 2012.

41. CHIARANTINI L., RIMONDI V., BENVENUTI M., BeUTEL M.W., COSTAGLIOLA P., GONNELli C., PAOLIERI M. Black pine (Pinus nigra) barks as biomonitors of airborne mercury pollution. Sci. Total Environ. 569, 105, 2016.

42. STAXÄNG B. Acidification of bark of some deciduous trees. Oikos. 20, 224, 1969.

43. HÄRTEL O., GRILL D. Die Leitfähigkeit von Fichtenborken - Extrakten als empfindlicher Indikator für Luftverunreinigungen. Eur. J. Forest Pathol. 2 (4), 205, 1972 [In German].

44. POIKOLAINEN J. Sulphur and heavy metal concentrations in Scots pine bark in northern Finland and the Kola Peninsula. Water Air Soil Poll. 93 (1-4), 395, 1997.

45. STEINDOR K., PALOWSKIB., GÓRAS P., NADGÓRSKASOCHAA. Assessment of bark reaction of select tree species as an indicator of acid gaseous pollution. Pol. J. Environ. Stud. 20 (3), 619, 2011.

46. LÖTSCHERT W., KÖHM H.J. Characteristics of tree bark as an indicator in high- immission areas. Oecologia. 27 (1), 47, 1977.

47. LÖTSCHERT W. Immissions analysen im Raum Frankfurt unter Verwendung pflan- zlicher Biodinidkatoren. Verh. Ges. Ökol. 11, 277, 1983 [In German].

48. YILMAZ R., SAKCALI S., YARCI C., AKSOY A., OZTURK M. Use of Aesculus hipp.ocastanum L. as a 
biomonitor of heavy metal pollution. Pak. J. Bot. 38 (5), 1519, 2006.

49. OLAJIRE A.A., AYODELE E.T. Study of atmospheric pollution levels by trace elements analysis of tree bark and leaves. B. Chem. Soc. Ethiopia. 17 (1), 11, 2003.

50. WEN S., YANG F., LI J.G., GONG Y., ZHANG X.L., HUI Y., WU Y.N., ZHAO Y.F., XU Y. Polychlorinated dibenzop-dioxin and dibenzofurans (PCDD/Fs), polybrominated diphenyl ethers (PBDEs), and polychlorinated biphenyls (PCBs) monitored by tree bark in an E- waste recycling area. Chemosphere. 74 (7), 981, 2009.

51. SAMECKA-CYMERMAN A., KOLON K., KEMPERS A.J. Taxus baccata as a bioindicator of urban environmental pollution. Pol. J. Environ. Stud. 20 (4), 1021, 2011.

52. GUÉGUEN F., STILlE P., GEAGEA M.L., BOUTIN R. Atmospheric pollution in an urban environment by tree bark biomonitoring- Part I: Trace element analysis. Chemosphere. 86 (10), 1013, 2012.

53. GUÉGUEN F., STILlE P., GEAGEA M. L., PERRONE T., CHABAUX F. Atmospheric pollution in an urban environment by tree bark biomonitoring- Part II: Sr, Nd and $\mathrm{Pb}$ isotopic tracing. Chemosphere. 86 (6), 641, 2012.

54. BARBEŞ L., BĂRBULESCU A., RĂDULESCU C., STIHI C., CHELARESCU E.D. Determination of heavy metals in leaves and bark of Populus nigra L. by atomic absorption spectrometry. Rom. Rep. Phys. 66 (3), 877, 2014.

55. CUTILLAS- BARREIRO L., ANSIAS- MANSO L., FERNÁNDEZ- CALVIÑO D., ARIAS- ESTÉVEZ M., NÓVOA- MUÑOZ J.C., FERNÁNDEZ- SANJURJO M.J., ALVARES- RODRIGUEZ E., NÚÑEZ- DELGADO A. Pine bark as bio-adsorbent for $\mathrm{Cd}, \mathrm{Cu}, \mathrm{Ni}, \mathrm{Pb}$ and $\mathrm{Zn}$ : Batch-type and stirred flow chamber experiments. J. Environ. Manage. 144, 258, 2014.

56. GUPTA J., GUPTA A., GUPTA A.K. Determination of trace metals in the stem bark of Moringa oleifera Lam. Int. J. Chem. Stud. 2 (4), 39, 2014.

57. ČEKSTERE G., LAIVINŠ M., OSVALDE A. Chemical Composition of Scots Pine Bark as a Bioindicator of Environmental Quality in Riga, Latvia. Proceedings of the Latvian Academy of Sciences. Section B: Natural, Exact, and Applied Sciences. 69 (3), (696), 87, 2015.

58. WANG T., YU J., HAN S., WANG Y., JIANG G. Levels of short chain chlorinated paraffins in pine needles and bark and their vegetation- air partitioning in urban areas. Environ. Pollut. 196, 309, 2015.

59. MANDIWANA K.L., RESANE T., PANICHEV N., NGOBENI P. The application of tree bark as bio- indicator for the assessment of $\mathrm{Cr}(\mathrm{VI})$ in air pollution. J. Hazard. Mater. 137 (2), 1241, 2006.

60. MOREIRA T.C.L., DE OLIVEIRA R.C., AMATO L.F.L., KANG C.M., SALDIVA P.H.N., SAIKI M. Intra- urban biomonitoring: Source apportionment using tree barks to identify air pollution sources. Environ. Int. 91, 271, 2016.

61. BELLIS D.J., SATAKE K., TSUNODA K., MCLEOD C.W. Environmental monitoring of historical change in arsenic deposition with tree bark pockets. J. Environ. Monitor. 5 (4), 671, 2003

62. WOLTERBEEK H.T., KUIK P., VERBURG T.G., WAMELINK G.W.W., VAN DOBBEN H. Relations between sulphate, ammonia, nitrate, acidity and trace element concentrations in tree bark in the Netherlands. Environ. Monit. Assess. 40 (2), 185, 1996.

63. SCHULZ H., POPP P., HUHN G., STÄRK H.J., SCHÜÜRMANN G. Biomonitoring of airborne inorganic and organic pollutants by means of pine tree barks. I. Temporal and spatial variations. Biomonitoring of atmospheric pollution (with emphasis on trace elements)BioMAP. Proceedings of an international workshop organized by the International Atomic Energy Agency in cooperation with the Instituto Tecnologico e Nuclear and held in Lisbon, Portugal, 21-24 September 1997. 149, Austria, 2000.

64. SCHULZ H., SCHULZ U., HUHN G., SCHÜRMANN G. Biomonitoring of airborne inorganic and organic pollutants by means of pine tree barks. II. Deposition types and impact levels. Biomonitoring of Atmospheric Pollution. Biomonitoring of atmospheric pollution (with emphasis on trace elements)- BioMAP. Proceedings of an international workshop organized by the International Atomic Energy Agency in co-operation with the Instituto Tecnologico e Nuclear and held in Lisbon, Portugal, 21-24 September 1997. 159, Austria, 2000.

65. PACHECO A.M.G., FREITAS M.C., BARROS L.I.C., FIGUEIRA R. Investigating tree bark as an air- pollution biomonitor by means of neutron activation analysis. J. Radioanal. Nucl. Chem. 249 (2), 327, 2001.

66. PACHECO A.M.G., BARROS L.I.C., FREITAS M.C., REIS M.A., HIPÓLITO C., OLIVEIRA O.R. An evaluation of olive-tree bark for the biological monitoring of airborne trace-elements at ground level. Environ. Pollut. 120 (1), 79, 2002.

67. PACHECO A.M.G., FREITAS M.C., BAPTISTA M.S., VASCONCELOS M.T.S.D., CABRAL J.P. Elemental levels in tree-bark and epiphytic-lichen transplants at a mixed environment in mainland Portugal, and comparisons with an in situ lichen. Environ. Pollut. 151 (2), 326, 2008.

68. SAARELA K.E., HARJU L., LILL J.O., HESELIUS S.J., RAJANDER J., LINDROOS A. Quantitative elemental analysis of dry- ashed bark and wood samples of birch, spruce and pine from south- western Finland using PIXE. Acta Acad. Abo. Ser. B. 65 (4), 1, 2005.

69. PATRICK G.J., FARMER J.G. A lead isotopic assessment of tree bark as a biomonitor of contemporary atmospheric lead. Sci. Total Environ. 388 (1), 343, 2007.

70. COSMA C., IURIAN A.R., INCZE R., KOVACS T., ŽUNIĆ Z.S. The use of tree bark as long term biomonitor of ${ }^{137} \mathrm{Cs}$ deposition. J. Environ. Radioactiv. 153, 126, 2016.

71. HITES R.A. Polybrominated diphenyl ethers in the environment and in people: a meta-analysis of concentrations. Environ. Sci. Technol. 38 (4), p45, 2004.

72. WANG Q., ZHAO Y., YAN D., YANG L., LI Z., HUANG B. Historical records of airborne polycyclic aromatic hydrocarbons by analyzing dated corks of the bark pocket in a Longpetiole Beech tree. Environ. Sci. Technol. 38 (18), 4739, 2004

73. HERMANSON M.H., JOHNSON G.W. Polychlorinated biphenyls in tree bark near a former manufacturing plant in Anniston, Alabama. Chemosphere. 68 (1), 191, 2007.

74. ORECCHIO S., GIANGUZZA A., CULOTTA L. Absorption of polycyclic aromatic hydrocarbons by Pinus bark: analytical method and use for environmental pollution monitoring in the Palermo area (Sicily, Italy). Environ. Res. 107 (3), 371, 2008

75. ZHAO Y., YANG L., WANG Q. Modeling persistent organic pollutant (POP) partitioning between tree bark and air and its app.lication to spatial monitoring of atmospheric POPs in mainland China. Environ. Sci. Technol. 42 (16), 6046, 2008.

76. FALAY E.O., TUNA G., ALTIOK H., KARA M., DUMANOGLU Y., BAYRAM A., ODABASI M. Spatial variation of polycyclic aromatic hydrocarbons (PAHs) in air, soil and tree components in Iskenderun industrial region, Turkey. Int. J. Chem. Environ. Biol. Sci. 1 (2), 263, 2013. 
77. ZHOU L., DONG L., HUANG Y., SHI S., ZHANG L., ZHANG X., YANG W., LI L. Spatial distribution and source app.ortionment of polycyclic aromatic hydrocarbons (PAHs) in Camphor (Cinnamomum camphora) tree bark from Southern Jiangsu, China. Chemosphere. 107, 297, 2014.

78. ZHOU L., DONG L., HUANG Y., SHI S., ZHANG L., ZHANG X., YANG W. Tree bark as a biomonitor for the determination of polychlorinated biphenyls and polybrominated diphenyl ethers from Southern Jiangsu, China: levels, distribution, and possible sources. Environ. Monitor. Assess. 187 (9), 1, 2015.

79. HERMANSON, M.H., JOHNSON, G.W. Polychlorinated dibenzofurans and dibenzo-p-dioxin in tree bark from an industrialized area: What the 2, 3, 7, 8-Cl substituted congeners tell us, and what is missing. J. Environ. Prot. 7 (3), 351, 2016.

80. LI Q., LU Y., JIN J., LI G., LI P., HE C., WANG Y. Comparison of using polyurethane foam passive samplers and tree bark samples from Western China to determine atmospheric organochlorine pesticide. J. Environ. Sci. 41, 90, 2016.
81. RAUERT C., HARNER T. A preliminary investigation into the use of Red Pine (Pinus resinosa) tree cores as historic passive samplers of POPs in outdoor air. Atmos. Environ. 140, 514, 2016.

82. HERMANSON M.H., HANN R., JOHNSON G.W. Polychlorinated biphenyls in tree bark near former manufacturing and incineration facilities in Sauget, Illinois, USA. Environ. Sci. Technol. 50 (12), 6207, 2016.

83. YUAN H., JIN J., BAI Y., LI Q., WANG Y., HU J. Concentrations and distributions of polybrominated diphenyl ethers and novel brominated flame retardants in tree bark and human hair from Yunnan Province, China. Chemosphere. 154, 319, 2016.

84. SALAMOVA A., HITES R.A. Brominated and chlorinated flame retardants in tree bark from around the globe. Environ. Sci. Technol. 47 (1), 349, 2012.

85. HE C., JIN J., LI G., WANG Y. Exchange of organohalogen compounds between air and tree bark in the Yellow River region. Chemosphere. 153, 478, 2016. 